Article

\title{
Regime Switching Vine Copula Models for Global Equity and Volatility Indices
}

\author{
Holger Fink $^{1,2, *}$, Yulia Klimova ${ }^{3}$, Claudia Czado ${ }^{3}$ and Jakob Stöber ${ }^{3}$ \\ 1 Faculty Business Administration and International Finance, Nürtingen-Geislingen University, \\ Sigmaringer Strasse 14, 72622 Nürtingen, Germany \\ 2 Center for Quantitative Risk Analysis, Department of Statistics, Ludwig-Maximilians-Universität München, \\ Akademiestr. 1/I, 80799 Munich, Germany \\ 3 Department of Mathematics, Technische Universität München, Boltzmannstraße 3, 85748 Garching, \\ Germany; yulia.klimova@gmx.de (Y.K.); cczado@ma.tum.de (C.C.); stoeber@ma.tum.de (J.S.) \\ * Correspondence: holger.fink@hfwu.de; Tel.: +49-7022-904-8357
}

Academic Editor: Jean-David Fermanian

Received: 30 June 2016; Accepted: 20 December 2016; Published: 4 January 2017

\begin{abstract}
For nearly every major stock market there exist equity and implied volatility indices. These play important roles within finance: be it as a benchmark, a measure of general uncertainty or a way of investing or hedging. It is well known in the academic literature that correlations and higher moments between different indices tend to vary in time. However, to the best of our knowledge, no one has yet considered a global setup including both equity and implied volatility indices of various continents, and allowing for a changing dependence structure. We aim to close this gap by applying Markov-switching $R$-vine models to investigate the existence of different, global dependence regimes. In particular, we identify times of "normal" and "abnormal" states within a data set consisting of North-American, European and Asian indices. Our results confirm the existence of joint points in a time at which global regime switching between two different $R$-vine structures takes place.
\end{abstract}

Keywords: regular vine copulas; Markov switching; implied volatility index; equity index; global dependence regimes

JEL Classification: C58; C52; C10; G10

\section{Introduction}

Based on the early work of [1,2], the Chicago Board Options Exchange (CBOE) started calculating its implied volatility index VIX, today well-known as a "fear gauge", back in 1993 . Thereby, they introduced not only a new asset class but also the first general index for market uncertainty. Early academic work therefore focused mainly on the relationship between the VIX and realized volatility (cf. [3-5]) and on pricing volatility derivatives (cf. [6]).

Over the years, other global exchanges followed suit leading to today's availability of volatility indices for nearly every major equity gauge. Accordingly, practical applications and academic research have broadened as well. For once, volatility indices can provide additional information for pricing equity options (cf. [7,8]). From a portfolio management perspective, similar to the well-known leverage effect initially discussed by [9], especially the usually observed asymmetric and negative dependence between implied volatility and equity indices can be useful for hedging purposes and risk management (cf. the recent work of [10] based on quantile regression or [11] on asymmetric volatility clustering). Additionally, implied volatility is in discussion to potentially have some predictive power on future returns as well (cf. [12,13]) even though such results may not be generally valid as recently shown by [14] and therein 
for currency carry strategies or [15] for fixed income markets. Finally, all these potential applications justify research on the modeling and prediction of volatility indices themselves as well (cf. [16,17]).

In this paper, however, we will contribute to a more macro-type application. As shown by the financial crisis back in 2007/2008 or the very recent sell-off in global equity markets in early 2016, major shifts in market sentiment are often not restricted to some area of the world but seem to quickly spread globally. Various researchers (as [18-23]) discussed in the past that, for such structural breaks within the dependencies between equity markets, (implied) volatility might play an important role, as well.

From a mathematical point of view, for such global analyses one faces the challenge of statistically describing higher-dimensional dependencies, which is usually tackled by either multivariate ARMA-GARCH setups or copula models. While the first ones might provide a fast and straightforward approach to analyze smaller data sets, parameters increase rather drastically for higher dimensions. Copulas on the other hand provide a neat way to circumvent such issues and therefore have found various applications in finance, e.g., in portfolio management (cf. [24] for a recent application or [25] for a good overview) or derivative pricing (cf. [26,27]). Several studies in the past invoked copula constructions to investigate either market dependencies, contagion or spillover effects as well, cf. [28-31].

However, to the best of our knowledge, there is no detailed analysis about the common dependence structure of global equity and implied volatility indices-a gap we are aiming to close in the present paper. Salazar and $\mathrm{Ng}$ [32] investigated a non-parametric tail dependence estimator but focused only on bivariate pairs, while Kasch and Caporin [33] only considered global equity indices based on a variation of the DCC-MGARCH model. Most closely related to our work is probably Peng and Ng [19] who applied a dynamic mixed copula approach with a focus on tail dependencies to analyze contagion effects between stock index movements and showed that financial shocks have a stronger effect on the relationship between volatility indices as on the one between equities. Additionally, they find that (asymmetric) tail dependencies tend to change and increase in post-crisis periods.

Building on the results of [19] but going a step further, we will additionally incorporate potential regime switches in our analysis to account for structural breaks. The general idea of such setups based on Markov chains was introduced by [34] and has recently been linked with copula models as well (cf. [35-38]). Especially the approach based on regular vine copulas of [38] is suited for our multivariate data set and we shall therefore build our analysis on their model setup. However, while they focused on modeling price returns - of FX rates to be precise-we shall include volatility indices as well. To be clear, the present paper does not aim to extend [38] from a theoretical perspective but invokes the method presented in there to fulfill our empirical goals. In particular, we are interested in a Markov model with two states which we shall call the "normal" and "abnormal" regime. We are deliberately avoiding the usually applied wording of "non-crisis" and "crisis" as it is ex-ante not clear how our two states should look like. In fact, we will show that an asymmetric, high tail dependence relationship between equity and volatility is rather the "normal" state than a "crisis".

The remainder of the paper is structured as follows. Section 2 presents our data set and discusses the filtering of each univariate index return series. Section 3 will briefly review the construction of $R$-vine-copulas (readers experienced in this field can skip Section 3.1). Furthermore, the new concept of quarter tail dependence (which is used to identify our two regimes later on) will be motivated and introduced. In Section 4 we provide a first, static application to our equity and volatility data, identifying plausible $R$-vine structures globally and for each continent individually. Section 5 summarizes the necessary preliminaries and results for regime-switching $R$-vine copulas from [38]. Afterwards, in Section 6, we carry out an extensive rolling window analysis to determine suitable copula families and motivate the classification of our "normal" and "abnormal" regimes. Different Markov-switching models are estimated, discussed and compared to their static counterparts. A summary closes the paper. All estimated models are summarized in the Appendix A.

All calculations have been performed with the statistical software package R. For the marginal models to be used in Section 2, we applied the function ugarchfit from the R-package rugarch by [39]. All vine copulas computations, such as model selection, likelihood calculation and parameter 
estimation, are carried out via the R package VineCopula by [40]. The fitting of the Markov-switching $R$-vine copula models is based on the code of [38].

\section{Data Description and Filtering}

The present paper considers eleven indices from three major regions: Asia, Europe and the USA. In total, we have five equity indices as well as their corresponding implied volatility indices (cf. Table 1). Additionally, one commodity index is taken into account: the Bloomberg Commodity ex-Agriculture and Livestock Index which is calculated mainly via futures trading in New York, Chicago and London. The considered time period covers roughly 13 years, starting in particular on 1 January 2002 and ending on 27 February 2015. Excluding non-trading days, this results in 3434 observations of daily closing prices in the respective domestic currency. The data source is Bloomberg and the absolute levels are depicted in Figure 1.

Table 1. Considered indices separated by regions.

\begin{tabular}{ccc}
\hline Shortcut & Index Description & Currency \\
\hline \multicolumn{4}{c}{ Asia } \\
NKY & Nikkei-225 Stock Average Index & JPY \\
VNKY & Hang Seng Index & JPY \\
HSI & Europe & HKD \\
VHSI & Implied Volatility Index of the HSI & HKD \\
\hline \multicolumn{4}{c}{} \\
\hline DAX & Deutscher Aktien Index (German Stock Index) & EUR \\
VDAX & Implied Volatility Index of the DAX & EUR \\
SX5E & Euro Stoxx 50 Index & EUR \\
VSX5E & Implied Volatility Index of the SX5E & EUR \\
\hline \multicolumn{4}{c}{ USA } & \\
\hline SPX & Standard and Poor's 500 Index & USD \\
VIX & Implied Volatility Index of the SPX & USD \\
BBC & Bloomberg Commodity ex-Agriculture \& Livestock Index & USD \\
\hline
\end{tabular}
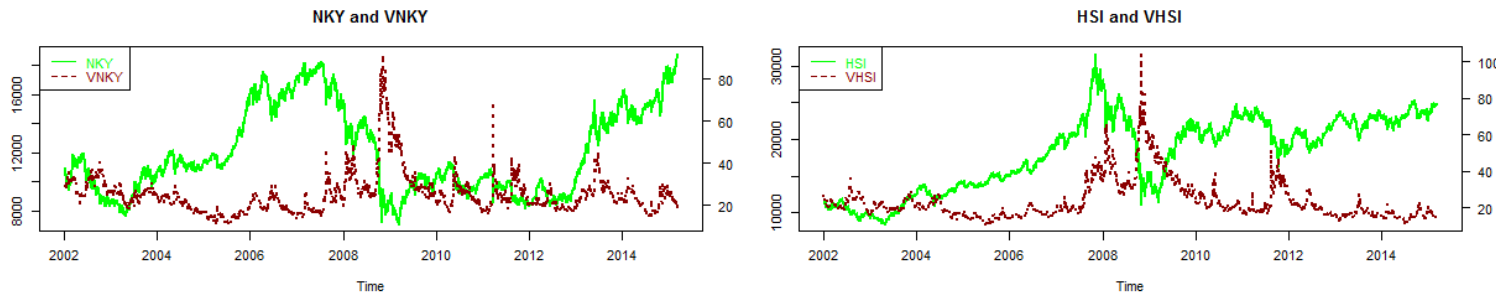

DAX and VDAX

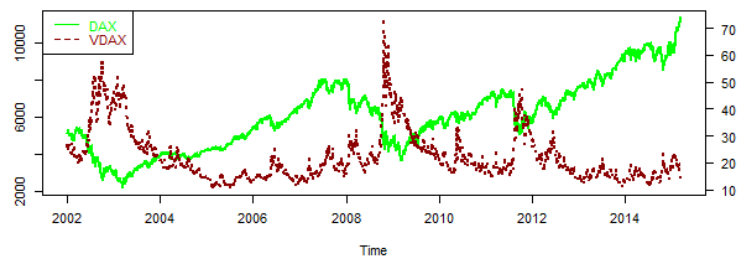

SX5E and VSX5E

SPX and VIX
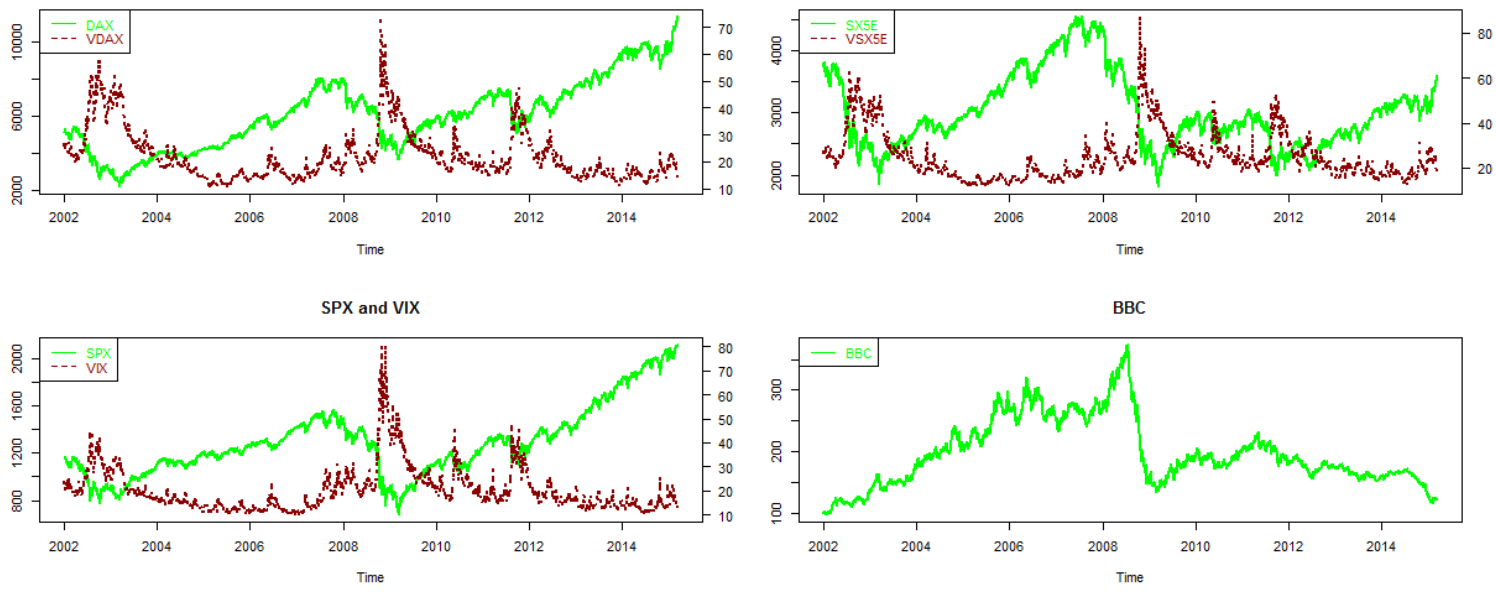

Figure 1. Absolute levels of the considered time series. 
We are aware of the time asynchronicity when dealing with closing prices of various continents. In fact, when measuring correlations, this issue can already be of significance for stocks trading at the same exchange and having just stale prices (cf. [41,42]). In particular, for the dependence structure of equity indices (measured via daily returns) it has been shown that considering liquid, internationally available exchange-traded funds (ETFs) can lead to different results as when analyzing the indices themselves (cf. [43-45] and therein). However, one does not have this option for volatility indices as there are no suitable ETFs tracking these. The existing ETFs are usually just rolling futures on the indices which is on a medium or longer time horizon a highly inefficient investment strategy due to the steep term structure of volatility futures.

Although lowering the observation frequency from daily to weekly returns (as has, e.g., been applied by [46] in a similar study) can mitigate asynchronicity effects, it might also obscure the potential dependence regimes too much.

Most suitable in our context would be the method proposed by [47] which basically "synchronizes" returns via a multivariate GARCH approach. However, it has been shown that the results of such a synchronization process (in terms of conditional correlations) are also highly model dependent (cf. [43] for a comparison of various approaches).

Similar to the argument in [48], our main objective is to describe and identify dependence regimes for the in-sample behavior of the present asynchronous index series. Therefore we shall work with the drawback of time asynchronicity for now. However, we want to stress that before one uses our results for applications like value-at-risk forecasting, the potential impact of return synchronization has to be analyzed in full detail, e.g., as carried out by [49] for their synchronous CCC-GARCH model.

Considering now the present daily returns, in a first step, we filter the marginal time series and transform them to uniformly distributed data on $[0,1]$ using ARMA-GARCH and ARMA-EGARCH models to account for features like time-changing volatility and clustering effects. In particular, we shall follow the procedure outlined in [50] (who considered a similar but smaller data set) and choose the respective lags, GARCH-setup and error distribution in terms of log-likelihood, AIC, BIC, the $p$-value of the Ljung-Box test and a graphical evaluation of $q q$-plots. The selected setups can be found in Table 2. The transformation of the obtained standardized residuals to uniformly distributed data is done by using the probability integral transform: Let $Z$ be a random variable with distribution function $F_{Z}$, then $U:=F_{Z}(Z)$ is uniformly distributed on $[0,1]$. The obtained transformed return series are now used in the following.

Table 2. The selected models and distribution of innovations for the marginal time series where GHYP stands for the generalized hyperbolic distribution.

\begin{tabular}{clc}
\hline Index & \multicolumn{1}{c}{ Marginal Model } & Distribution of Innovations \\
\hline NKY & ARMA $(3,2)-G A R C H(1,1)$ & GHYP \\
VNKY & ARMA $(3,0)-G A R C H(1,1)$ & GHYP \\
HSI & ARMA $(0,0)-G A R C H(1,1)$ & GHYP \\
VHSI & ARMA $(4,2)-G A R C H(1,1)$ & GHYP \\
\hline DAX & ARMA $(0,0)-G A R C H(1,1)$ & GHYP \\
VDAX & ARMA $(0,0)-E G A R C H(1,1)$ & GHYP \\
SX5E & ARMA $(1,0)-G A R C H(1,1)$ & GHYP \\
VSX5E & ARMA $(3,0)-G A R C H(1,1)$ & GHYP \\
\hline SPX & ARMA $(3,0)-G A R C H(1,1)$ & GHYP \\
VIX & ARMA $(3,0)-G A R C H(1,1)$ & GHYP \\
BBC & ARMA $(0,0)-G A R C H(1,1)$ & GHYP \\
\hline
\end{tabular}




\section{Copula Models without Regime Switches}

In the following section we shall briefly review the main concepts of (regular vine) copulas, the core tool of our upcoming investigations, starting with the very general definition. Additionally, we introduce a new measure called quarter tail dependence to capture tail asymmetries within our equity and volatility data set. Readers experienced in $R$-vine-copulas can skip the next subsection and continue with Section 3.2.

\subsection{Regular Vine Copulas}

For more details on (vine) copulas, we refer the interested reader to [51-56]. The general definition follows.

Definition 1. Let $d \geq 2$. A d-dimensional cumulative distribution function on $[0,1]^{d}$ with uniform, univariate marginal distributions is called a copula. If the derivative of $C$ exists, its copula density is given by

$$
c\left(u_{1}, \ldots, u_{d}\right)=\frac{\partial^{d}}{\partial u_{1} \cdots \partial u_{d}} C\left(u_{1}, \ldots, u_{d}\right)
$$

The seminal theorem of [57] clarifies that for every multivariate distribution, a suitable copula exists.

Theorem 1 (Sklar's theorem). Let $d \geq 2$ and $X=\left(X_{1}, \ldots, X_{d}\right)$ be a random vector with margins $F_{1}, \ldots, F_{d}$ and a joint distribution function $F$. Then there exists a copula $C$, such that

$$
F\left(x_{1}, \ldots, x_{d}\right)=C\left(F_{1}\left(x_{1}\right), \ldots, F_{d}\left(x_{d}\right)\right) \quad \forall x=\left(x_{1}, \ldots, x_{d}\right)^{T} \in \mathbb{R}^{d} .
$$

If $F_{1}, \ldots, F_{d}$ are continuous, then the copula $C$ is unique. Conversely, if $F_{1}, \ldots, F_{d}$ are univariate distribution functions and $C$ is a d-dimensional copula, then $F$ defined via (2) is a d-dimensional distribution function.

Straightforward examples which can be represented analytically even in higher dimensions are the classical Gauss or Student- $t$ copulas. However, for the purpose of modeling financial data, these might not always be a sensible choice: The Gaussian copula is not able capture tail dependence, while the Student- $t$ copula only allows for symmetric dependence in the lower and upper tails.

An elegant way of constructing more general multivariate distributions through conditioning using bivariate copulas was first introduced by [58,59] based on earlier work of [60] (see also [55] for a recent summary). The resulting so-called pair copula construction (PCC) which has been investigated in detail by $[61,62]$ can be neatly illustrated in three dimensions by the following example: Let $f$ be the continuous density function of a 3-dimensional distribution function $F$ with marginals $F_{1}, F_{2}$ and $F_{3}$. Then we can decompose

$$
\begin{aligned}
f\left(x_{1}, x_{2}, x_{3}\right) & =c_{13 ; 2}\left(F_{1 \mid 2}\left(x_{1} \mid x_{2}\right), F_{3 \mid 2}\left(x_{3} \mid x_{2}\right)\right) \\
& \times c_{23}\left(F_{2}\left(x_{2}\right), F_{3}\left(x_{3}\right)\right) c_{12}\left(F_{1}\left(x_{1}\right), F_{2}\left(x_{2}\right)\right) \\
& \times f_{3}\left(x_{3}\right) f_{2}\left(x_{2}\right) f_{1}\left(x_{1}\right),
\end{aligned}
$$

where $c_{13 ; 2}$ is the copula density associated with the distribution of $\left(X_{1}, X_{3}\right)$ given $X_{2}=x_{2}$. In the following, we invoke the usually imposed simplifying assumption which states that $c_{13 ; 2}$ is independent of $x_{2}$. For a detailed discussion of its implication and potential restrictiveness we refer to [63-65]. Also, the current non-simplified models are restricted to mostly three dimensions. In addition a dynamic formulation as we will propose allows to mitigate potential non-simplifying effects.

By repeated conditioning, the above outlined procedure can directly be generalized to higher dimensions. One has to keep in mind that decomposition (3) is generally not unique. However, 
using graph-theoretical fundamentals a certain choice can be illustrated graphically in a compact and clear way (cf. [58] and more generally [66]).

Definition 2. Let $d \geq 2$. A regular vine ( $R$-vine) is an ordered sequence of trees $\mathcal{V}=\left(T_{1}, \ldots, T_{d-1}\right)$ with $T_{i}=\left(N_{i}, E_{i}\right), i \in\{1, \ldots, d-1\}$, where $N_{i}$ is a set of nodes and $E_{i}$ a set of edges, such that

1. $N_{1}=\{1, \ldots, d\}$, i.e., the first tree has nodes $1, \ldots, d$,

2. for $i \in\{2, \ldots, d-1\}$ we have $N_{i}=E_{i-1}$, i.e., the nodes of $T_{i}$ are the edges of $T_{i-1}$,

3. if for $i \in\{1, \ldots, d-2\}$ two nodes of $T_{i+1}$ are connected, the corresponding edges in $T_{i}$ have a common node (proximity condition).

If on each tree, every node has maximally two edges, we speak of a drawable vine ( $D$-vine) while if on each tree there exists one node, that is connected to all other nodes, we call this a canonical vine (C-vine).

Coming back to our 3-dimensional example in (3), the corresponding $R$-vine describing this structure is depicted by Figure 2. In such cases, the corresponding copula $C$ is called a $R$-vine copula and analogously a D-vine copula or C-vine copula. Since we considered only 3 dimensions, Figure 2 actually shows both, a $D$ - and $C$-vine structure.

Tree 1

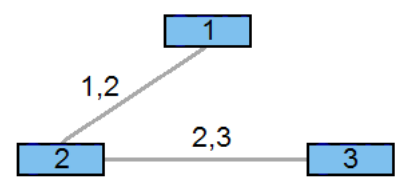

Tree 2

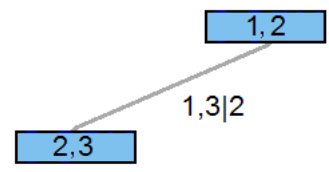

Figure 2. Graphical representation of decomposition (3). Relevant are the connections between the boxes (not their location).

Having outlined how multivariate copulas can be constructed using bivariate distributions, we still have to choose suitable two-dimensional copulas. In general, there are two major bivariate copula families: Elliptical and Archimedean. The first group contains the bivariate Gauss and Student- $t$ copulas while examples for the Archimedean ones are the Clayton, Gumbel, Frank or Joe copulas. A very neat overview is presented in [67]. In the following, we will consider Gauss and Student- $t$ copulas which are the most common bivariate models used in financial applications. Due to their property of allowing asymmetric tail dependence, we shall further focus on the Gumbel copula and its counter-clockwise rotations defined for $\theta \in[1, \infty)$ and $u, v, \in[0,1]$ via

$$
\begin{aligned}
C_{\text {Gumbel }}(u, v) & =\exp \left\{-\left[(-\log (u))^{\theta}+(-\log (v))^{\theta}\right]^{\frac{1}{\theta}}\right\} \\
C_{\text {Gumbel90 }}(u, v) & =v-C_{\text {Gumbel }}(1-u, v) \\
C_{\text {Gumbel180 }}(u, v) & =u+v-1+C_{\text {Gumbel }}(1-u, 1-v) \\
C_{\text {Gumbel270 }}(u, v) & =u-C_{\text {Gumbel }}(u, 1-v)
\end{aligned}
$$

We finish this subsection by recalling Kendall's $\tau$, the most widely used dependence measure in copula theory due to its property of being invariant with respect to strictly monotone transformations of the marginal distributions. Therefore the ARMA-GARCH filtering in Section 1 does not influence Kendall's $\tau$. In fact, if $C$ is the corresponding copula for a bivariate random vector $(X, Y)^{T}$, then we have

$$
\tau(X, Y)=4\left[\int_{[0,1]^{2}} C\left(u_{1}, u_{2}\right) d C\left(u_{1}, u_{2}\right)\right]-1 .
$$


For given sample data $\left(x_{1}, y_{1}\right)^{T}, \ldots\left(x_{n}, y_{n}\right)^{T}, n \geq 2$, an estimate of Kendall's $\tau$ is given by

$$
\hat{\tau}(X, Y)=\frac{N_{c}-N_{d}}{\sqrt{N_{c}+N_{d}+N_{X}} \cdot \sqrt{N_{c}+N_{d}+N_{Y}}} .
$$

where $N_{d}$ and $N_{c}$ are the numbers of discordant and concordant pairs, respectively, while $N_{X}\left(N_{Y}\right)$ denotes the amount of the pairs with $x_{i}=x_{j}\left(y_{i}=y_{j}\right)$ where $i, j \in\{1, \ldots, n\}, i \neq j$ ([53]). For a data set without ties, there are simpler formulas, as well. We shall call $\hat{\tau}(X, Y)$ the empirical Kendall's $\tau$.

\subsection{Quarter Tail Dependence}

As pointed out before, the main aim of this paper is to identify "normal" and "abnormal" dependence regimes within the relationship between equity and implied volatility indices. Usually, one can observe that in times of market turmoil especially larger negative returns of stocks show a high degree of dependence not present between similarly large positive returns. The same holds inversely true for the relationship between the right compared to the left tails of two volatility indices. Both effects can be captured by the well-known (bivariate) lower and upper tail dependence coefficients defined as follows:

Definition 3 (Tail dependence coefficients). For a copula $C$ of a random vector $(U, V)^{T}$ with marginal distribution functions $F_{1}$ and $F_{2}$, we define its upper and lower tail dependence coefficients (TDCs) via

$$
\begin{aligned}
& \lambda_{U}(C)=\lim _{u \nearrow 1} \mathbb{P}\left(V>F_{2}^{-1}(u) \mid U>F_{1}^{-1}(u)\right)=\lim _{u \nearrow 1} \frac{1-2 u+C(u, u)}{1-u}, \\
& \lambda_{L}(C)=\lim _{u \searrow 0} \mathbb{P}\left(V \leq F_{2}^{-1}(u) \mid U \leq F_{1}^{-1}(u)\right)=\lim _{u \searrow 0} \frac{C(u, u)}{u} .
\end{aligned}
$$

The measures $\lambda_{U}(C)$ and $\lambda_{L}(C)$ are able to capture (bivariate) relationships within the first and third quadrant of $[0,1]^{2}$. Thus, not every copula family has a non-zero upper or lower tail dependence, cf. Table 3. Consider, e.g., the Gumbel copula and its rotations: all four copulas describe different tail dependence properties. The upper tail dependence coefficient is non-zero only for the Gumbel copula while the lower tail dependence coefficient vanishes for all but Gumbel180.

Table 3. Tail dependence coefficients for different copula families. $\theta$ is the copula parameter for the Gumbel/Student- $t$ families and $v$ denotes the degrees of freedom of the Student- $t$ copula.

\begin{tabular}{ccc}
\hline Copula Family & $\lambda_{L}$ & $\lambda_{U}$ \\
\hline Gauss & - & - \\
Student- $t$ & $2 t_{v+1}\left(-\sqrt{v+1} \sqrt{\frac{1-\theta}{1+\theta}}\right)$ & $2 t_{v+1}\left(-\sqrt{v+1} \sqrt{\frac{1-\theta}{1+\theta}}\right)$ \\
Gumbel & - & $2-2^{1 / \theta}$ \\
Gumbel90 & - & - \\
Gumbel180 & $2-2^{1 / \theta}$ & - \\
Gumbel270 & - & - \\
\hline
\end{tabular}

However, when it comes to the asymmetric relationship between equity and volatility, we would expect Gumbel90 or Gumbel270 to be fitting better as the "normal" relation observed in financial markets is often the following: On the one side, turmoil in equity markets is usually accompanied by a steep rise in volatility levels. On the other side, booming markets tend to be associated with declining volatility even though this effect is usually not that strong. As shown above, this asymmetric relationship can not be captured by the lower or upper tail dependence coefficients.

Additionally, we are considering a commodity index, as well. In a time period of stagnating or declining global growth, which would be accompanied by low or negative equity and high volatility returns, one can expect that the demand for, e.g., industrial metals and energy tends to fall heavily. 
As consequence, the tail dependence for a commodity-volatility index pair should be analogues to an equity-volatility pair.

There are various ways to describe this kind of asymmetric dependence structure (cf. [32] who considered tail copulas). For our purposes, we shall slightly tweak the definition of the tail dependence coefficients to get suitable measures for the above described relationships:

Definition 4 (Quarter tail dependence). Let $(U, V)^{T}$ be a random vector with uniformly distributed marginals. We specifically assume, that $(U, V)^{T}$ are correspondingly transformed returns of equity $(E q)$, volatility (Vol) or commodity (Com) indices, using from now on the notation $U, V \in(E q, V o l, C m d)$. Furthermore let the copula $C$ of $(U, V)^{T}$ be either Gauss (Ga), Student- $t$ (St-t), Gumbel or one of its rotations (Gu, Gu90, Gu180 or Gu270). Then the quarter tail dependence (QTD) is defined by

$$
\lambda_{Q T D}(U, V, C)= \begin{cases}\lambda_{\text {QTD }}^{1}(C), & \text { if }(U, V)^{T}=(\text { Vol, Vol })^{T} \\ \lambda_{\text {QTD }}^{2}(C), & \text { if }(U, V)^{T} \in\left\{(E q, V o l)^{T},(C m d, V o l)^{T}\right\} \\ \lambda_{Q T D}^{3}(C), & \text { if }(U, V)^{T} \in\left\{(E q, E q)^{T},(C m d, E q)^{T},(E q, C m d)^{T}\right\} \\ \lambda_{Q T D}^{4}(C), & \text { if }(U, V)^{T} \in\left\{(\text { Vol }, E q)^{T},(\text { Vol }, C m d)^{T}\right\}\end{cases}
$$

where

$$
\begin{aligned}
\lambda_{\text {QTD }}^{1}(C) & =\lambda_{U}(C), \\
\lambda_{Q T D}^{2}(C) & = \begin{cases}\lambda_{U}(S t(-\theta, v)), & \text { if } C=S t(\theta, v) \text { with } \tau<0 \\
\lambda_{U}(G u(-\theta)), & \text { if } C=G u 90(\theta) \\
0, & \text { else, }\end{cases} \\
\lambda_{\text {QTD }}^{3}(C) & =\lambda_{L}(C), \\
\lambda_{\text {QTD }}^{4}(C) & = \begin{cases}\lambda_{U}(S t(-\theta, v)), & \text { if } C=S t(\theta, v) \text { with } \tau<0 \\
\lambda_{U}(G u(-\theta)), & \text { if } C=G u 270(\theta) \\
0, & \text { else, }\end{cases}
\end{aligned}
$$

where $\lambda_{L}$ and $\lambda_{U}$ denote the lower and upper tail dependence coefficients as stated in Definition 3 and given in Table 3 for the chosen copula families. Furthermore $\theta$ is the Gumbel/Student- $t$ copula parameter and $v$ denotes number of degrees of freedom in case of the Student-t copula.

As one can see, the (classical) upper tail dependence coefficient, which describes the dependence in the first quadrant, is used for the calculation of the QTD for a volatility-volatility pair. For an equity-equity as well as an equity-commodity pair we are interested in the third quadrant, which is measured using the lower tail dependence coefficient. Finally, an equity-volatility or a commodity-volatility relationship is located in the second and fourth quadrant and captured by $\lambda_{\mathrm{QTD}}^{2}(C)$ and $\lambda_{\mathrm{QTD}}^{4}(C)$. An illustration of the QTD-definition is given by Table 4 . 
Table 4. Quarter tail dependence $\lambda_{Q T D}(X, Y, C)$ for different index pairs and copula families: Gauss, Student- $t$ and Gumbel including rotations. The graphical example is based on contour plots of the Gumbel copula and its rotations with Kendall's $\tau$ of 0.3 and -0.3 respectively.

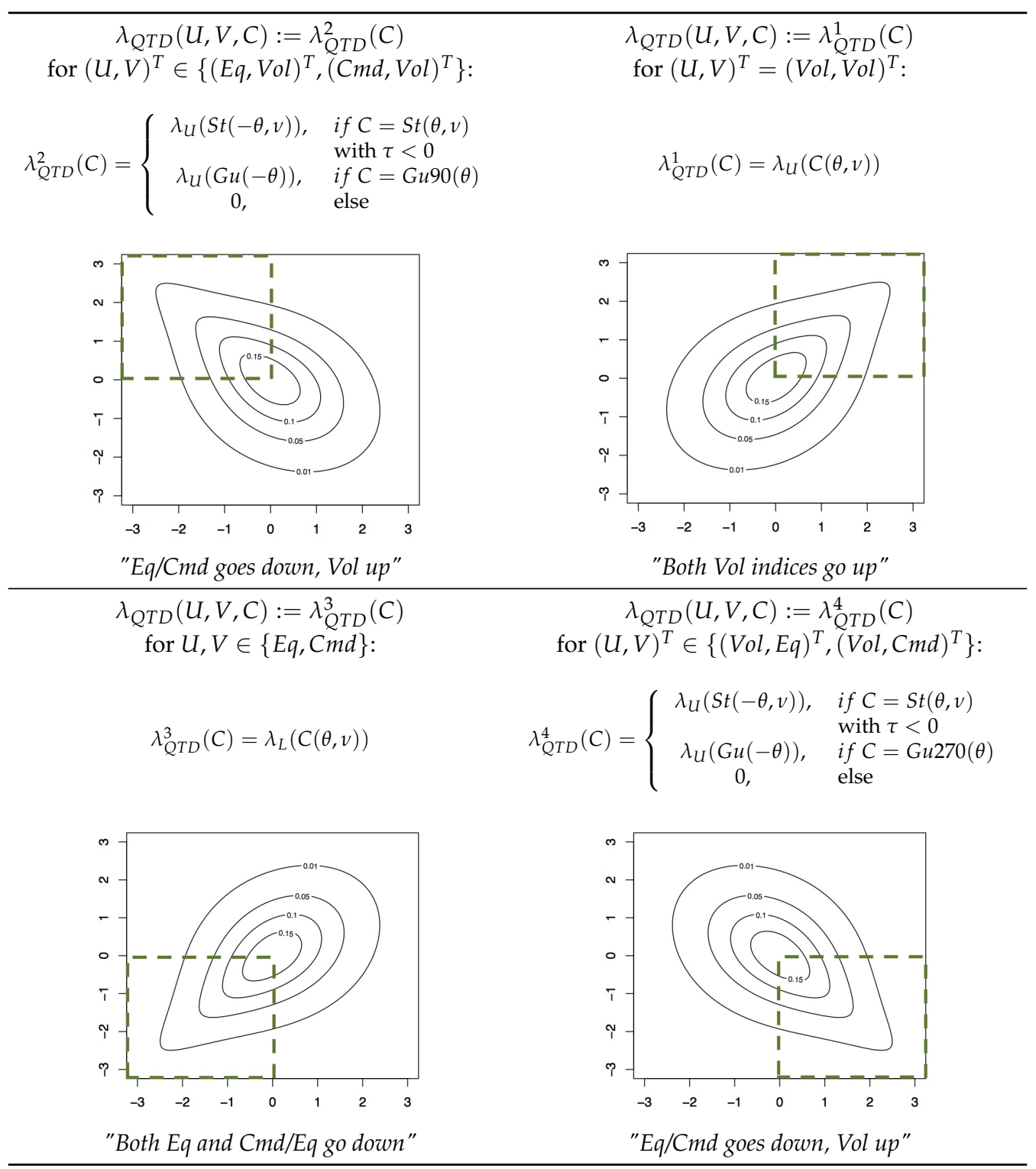

\section{Identifying Plausible $R$-Vine Tree Structures}

In this section we shall provide a first, static look, at our index data. Considering the filtered return series from Section 2, we firstly apply the $R$-vine structure selection technique of [68] (which is basically connecting those nodes with the highest dependence measured by Kendall's $\tau$ ), i.e., no restrictions on tree structure and chosen copula families (Gaussian (Ga), Student- $t$ (St- $t$ ) and Gumbel $(\mathrm{Gu}$ ) including all rotations (Gu90, Gu180 and Gu270)) are imposed. We shall call the resulting $R$-vine model (1-dependent) and its first two trees are depicted in Figure 3. The upcoming static global models with pre-defined tree structure will be denoted by " $(2-\ldots)$ " while we use " $(3-\ldots)$ " for the final regime switching models for whom the $R$-vine structures shall also be given. The wording "dependent" or "independent" shall indicate whether we allow for a potential dependence structure between continents or not. All models and estimated parameters are summarized in the Appendix A. 
Tree 1

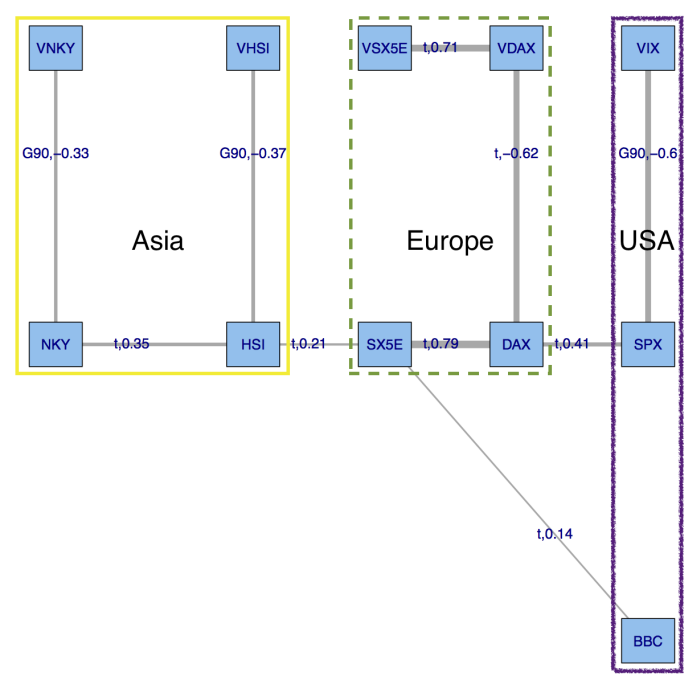

Tree 2

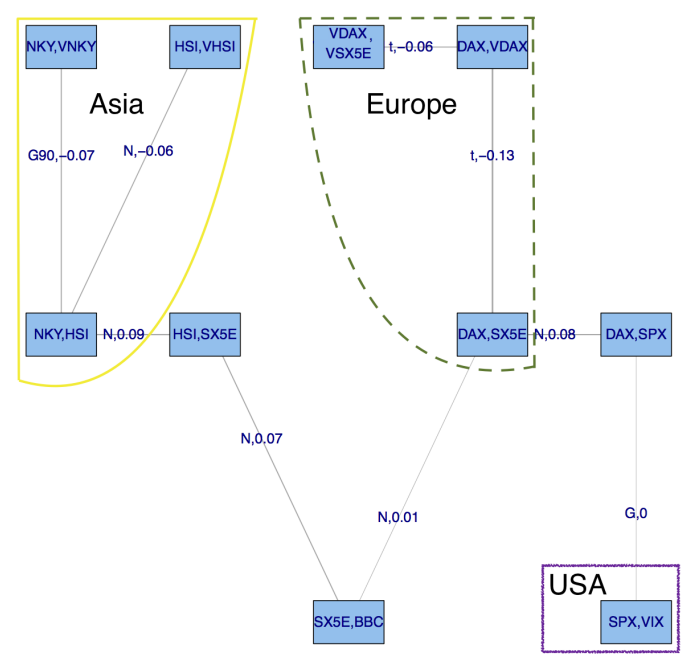

Figure 3. Model (1-dependent): first and second tree, corresponding bivariate copula families and values of Kendall's $\tau$.

As we can see, the equity indices connect directly with their corresponding volatility counterparts indicating a strong dependence structure between both. On a more macro scale, this also holds true for the considered geographical regions as indices from the same continent seem to "stick together"- the commodity index being the only exception which could be explained by the fact that BBC is a global index, with underlying futures prices determined in New York, Chicago and London. Additionally and as expected, Kendall's $\tau$ indicates a positive dependence between equity-equity and equity-commodity and a negative one for equity-volatility.

Apart from BBC being linked with an European index, the continents are mainly connected by two pairs of equity indices, namely HSI-SX5E and DAX-SPX, which can be neatly explained by considering global trading hours (cf. Table 5).

Table 5. International trading hours (ignoring potential lunchbreaks).

\begin{tabular}{cc}
\hline Stock Exchange & Opening-Closing Time (UTC) \\
\hline \multicolumn{2}{c}{ Asia } \\
\hline Tokyo Stock Exchange & $00: 00-06: 00$ \\
Hong Kong Stock Exchange & $01: 30-08: 00$ \\
\hline \multicolumn{2}{c}{ Europe } \\
\hline Frankfurt Stock Exchange (Xetra) & $08: 00-16: 30$ \\
Eurex Exchange (for SX5E index options) & $08: 00-16: 30$ \\
\hline USA \\
\hline New York Stock Exchange \\
\hline
\end{tabular}

Finally, the level of connection measured by Kendall's $\tau$ is seen to be lower on the second than on the first tree while the dependence structures within each region seem to be stronger than those between the continents. For the later analysis, it suggests a more detailed investigation of the behavior within and between continents. However, as the Markov-switching setup to be used later in the paper requires us to define the $R$-vine structures for both regimes upfront and since we would like to understand the behaviour of the individual regions, as well, we shall from now on work without selection algorithms and derive a suitable intra- and intercontinental dependence structure by hand.

Therefore, to prepare a potential (and plausible!) pre-defined tree structure for our regime switching setup, we start by investigating all three intra-regional dependencies separately. Only in 
a second step, we shall add a global tree structure. Starting with Asia, we consider four setups, namely Asia ${ }_{1}-\mathrm{Asia}_{4}$ depicted in Figure 4. The first two models, Asia ${ }_{1}$ and Asia 2 , are $D$-vines, in which the connections are either made through equities (NKY-HSI) or volatilities (VNKY-VSHI), respectively. In the $C$-vine model Asia 3 the central node is the equity index HSI at the Hong Kong stock exchange while in $\mathrm{Asia}_{4}$ this role is taken over by the Japanese NKY. From now on, for a given tree structure, estimation and copula selection is carried out based on maximum likelihood and AIC using the function RVineCopSelect out of the VineCopula package by [40].

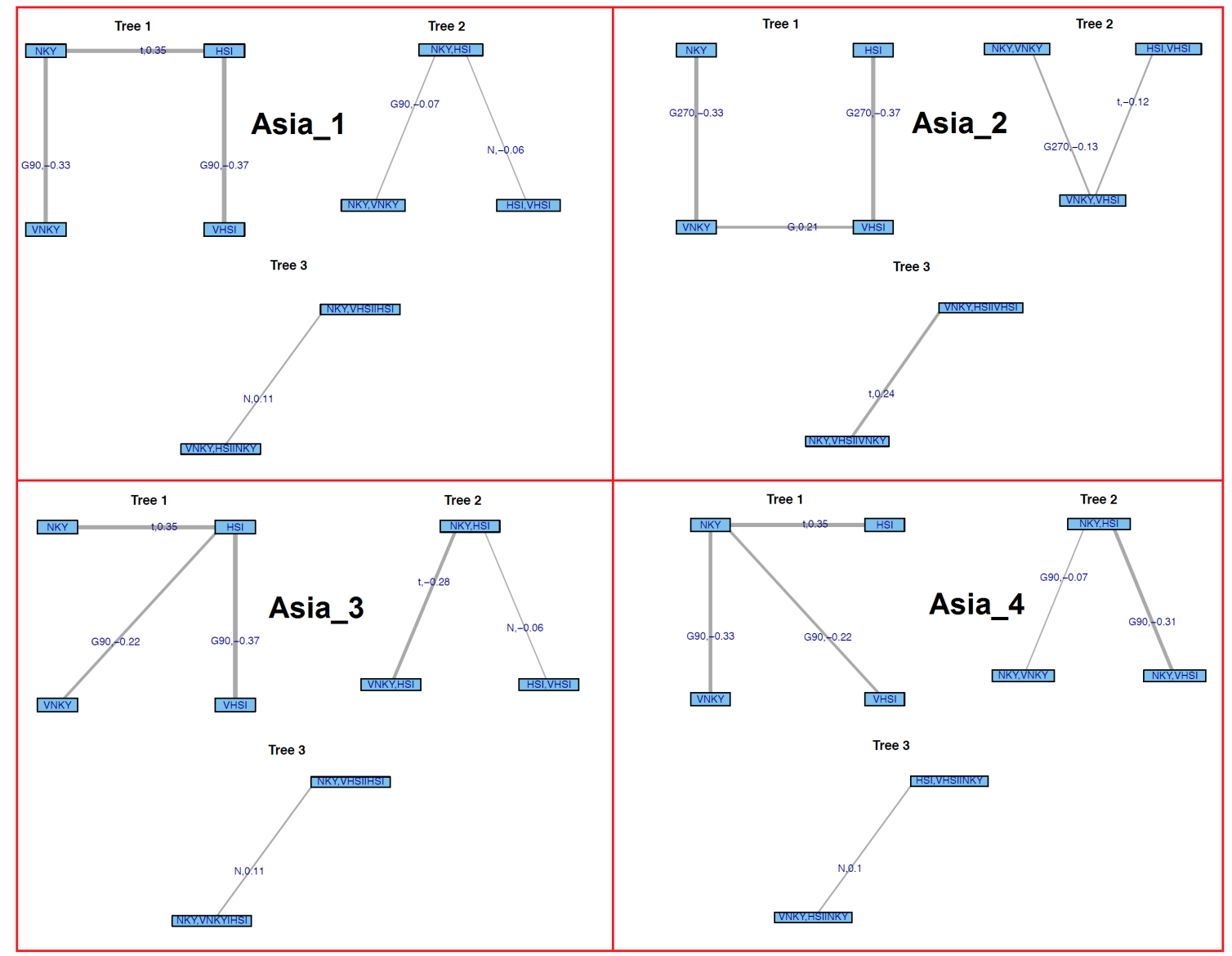

Figure 4. Tree structures for models Asia $1-\mathrm{Asia}_{4}$ and values of Kendall's $\tau$.

Europe $_{1}$ and Europe ${ }_{2}$ are built analogously to the ones in the Asian region. The central node of the $C$-vine Europe ${ }_{3}$ is chosen to be the broader European equity index SX5E. As the DAX is partially included in the SX5E, Europe 4 is taken to be another D-vine for which the "middle" nodes are the DAX and its implied volatility index VDAX. Another reason for this particular setup is that this structure was also chosen in (1-dependent) and we want to test whether this is optimal when Europe is analyzed separately. All models are depicted in Figure 5. 


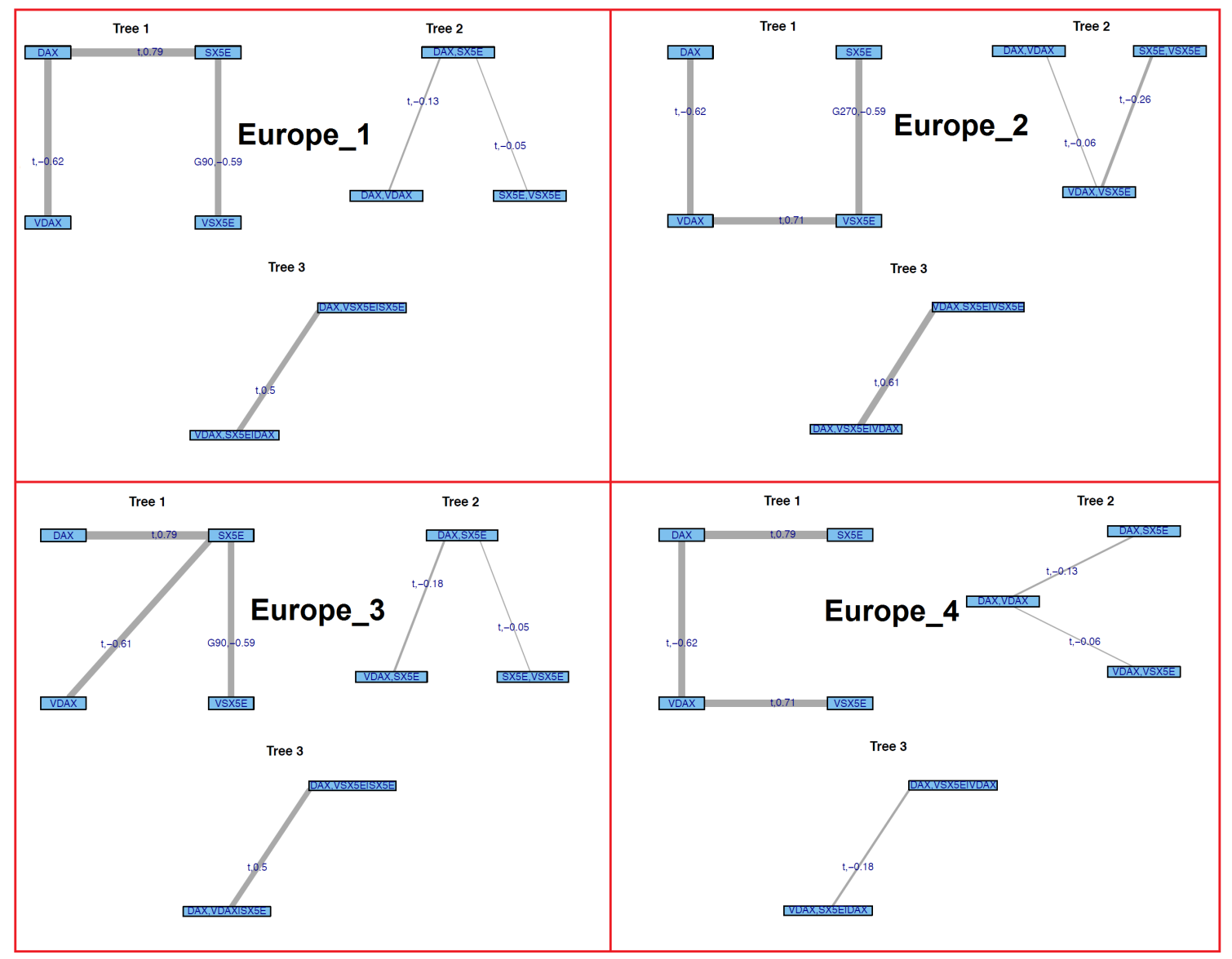

Figure 5. Tree structures for models Europe ${ }_{1}-$ Europe $_{4}$ and values of Kendall's $\tau$.

Finally, the considered structures for North-America, $\mathrm{USA}_{1}$ and USA 2 , are shown in Figure 6. Both are $C$-vine models where the central node in the first tree is either the SPX or the VIX.

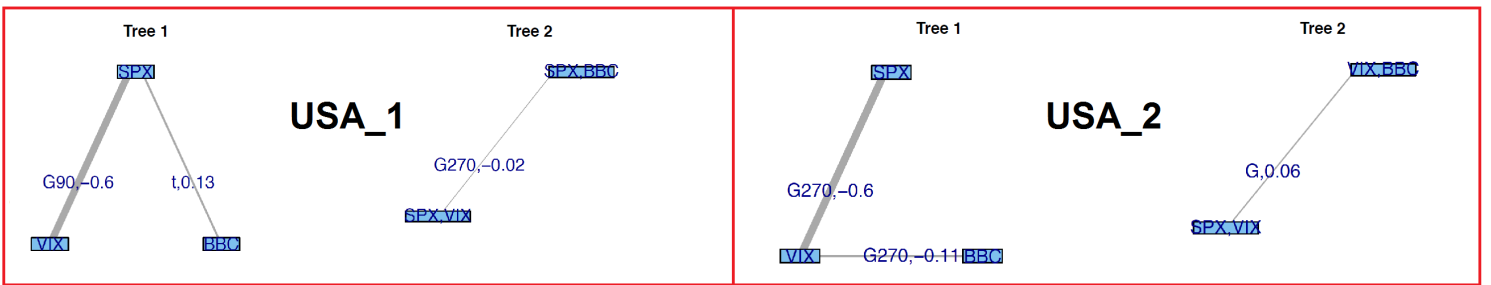

Figure 6. Tree structures for models $\mathrm{USA}_{1}-\mathrm{USA}_{2}$ and values of Kendall's $\tau$.

For all setups, the log-likelihood and information criteria are presented in Table 6. Generally, the $D$-vine models Asia ${ }_{1}$, Europe $_{1}$ and USA $_{1}$ deliver the highest log-likelihoods and the best AIC and BIC values as well.

We want to stress that, in general, as the $R$-vine decomposition is not unique, different structures with different parameters might lead to the same distribution. This occurs in the case when all pair copulas are Gaussian, bivariate $t$ - or Clayton copulas. In the $t$ - and Clayton case, however, further restrictions on the parameters for different trees have to be satisfied. In practice, these cases rarely occur and here the estimation leaves us with differing likelihoods for all considered setups (cf. Table 6). To ensure that our regimes (to be identified later in the paper) are really distinct, we will make use of empirical confidence bounds, as well. 
Table 6. Log-likelihood, AIC and BIC for the considered $R$-vine models Asia 1 -Asia 4 , Europe ${ }_{1}-$ Europe $_{4}$ and $\mathrm{USA}_{1}-\mathrm{USA}_{2}$ for the geographical regions. Combining the bold models leads to (2-independent).

\begin{tabular}{cccc}
\hline & Log-Likelihood & AIC & BIC \\
\hline Model Asia $_{1}$ & $\mathbf{2 0 2 7 . 6 1}$ & $-\mathbf{4 0 4 1 . 2 2}$ & $-\mathbf{3 9 9 8 . 2 4}$ \\
Model Asia $_{2}$ & 2014.13 & -4012.25 & -3963.12 \\
Model Asia $_{3}$ & 1997.10 & -3978.21 & -3929.08 \\
Model Asia $_{4}$ & 1973.17 & -3932.35 & -3889.36 \\
\hline Model Europe $_{1}$ & $\mathbf{8 9 7 3 . 4 8}$ & $\mathbf{- 1 7 , 9 2 4 . 9 5}$ & $-\mathbf{1 7 , 8 5 7 . 4 0}$ \\
Model Europe $_{2}$ & 8758.88 & $-17,495.76$ & $-17,428.21$ \\
Model Europe $_{3}$ & 8964.05 & $-17,906.11$ & $-17,838.55$ \\
Model Europe $_{4}$ & 8944.14 & $-17,864.27$ & $-17,790.58$ \\
\hline Model USA $_{1}$ & $\mathbf{2 0 1 7 . 6 3}$ & $-\mathbf{4 0 2 7 . 2 6}$ & $-\mathbf{4 0 0 2 . 6 9}$ \\
Model USA $_{2}$ & 2009.69 & -4013.37 & -3994.95 \\
\hline
\end{tabular}

Finally, we combine the previous results to set up a global model: Within each region, the structure of the first trees is inherited from Table 6 while the connections between continents are made via the same pairs as in (1-dependent), i.e., HSI-SX5E and DAX-SPX, resulting in model (2-dependent) depicted by Figure 7. In particular, in terms of log-likelihood, as well as AIC and BIC, the new tree structure seems to be slightly better than (1-dependent) as summarized by Table 7. Not very surprisingly, it also outperforms (2-independent) which is built from Asia ${ }_{1}$, Europe $_{1}$ and $\mathrm{USA}_{1}$ by assuming independent continents.

Tree 1

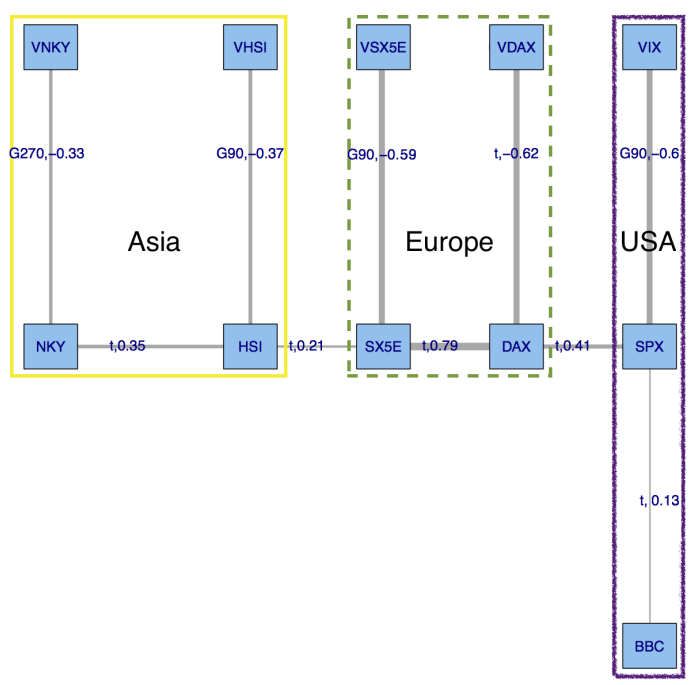

Tree 2

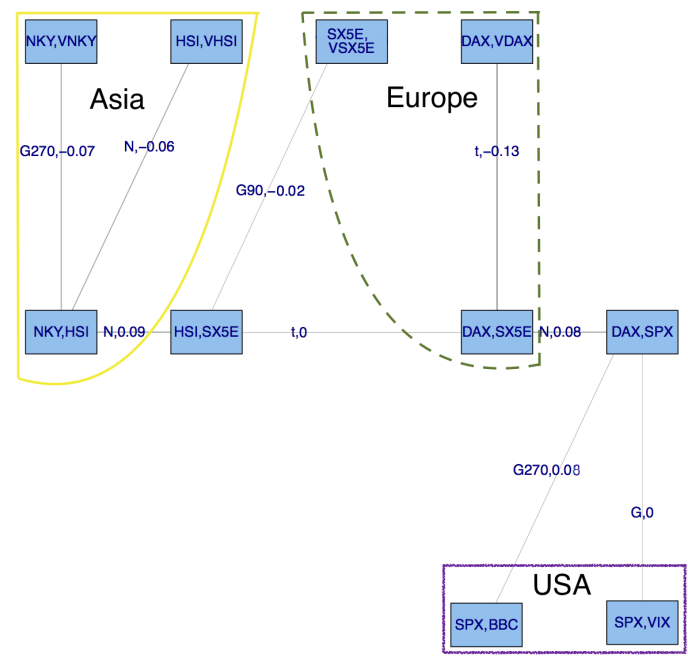

Figure 7. Model (2-dependent): first and second tree, corresponding bivariate copula families and values of Kendall's $\tau$.

Table 7. Log-likelihood, AIC and BIC for the considered $R$-vine models (1-dependent), (2-independent) and (2-dependent).

\begin{tabular}{cccc}
\hline & Log-Likelihood & AIC & BIC \\
\hline (1-dependent) & $14,578.12$ & $-29,010.24$ & $-28,561.94$ \\
(2-independent) & $13,018.72$ & $-25,993.44$ & $-25,858.33$ \\
(2-dependent) & $14,618.70$ & $-29,091.40$ & $-28,643.09$ \\
\hline
\end{tabular}




\section{Markov-Switching $R$-Vine Copula Models}

In this section we review the regime switching setup based on $R$-vine copulas from [38], which will later on be used to model potential changes within the dependence structure of our index data set. As we are in particular interested in describing a "normal" and an "abnormal" market state, we will for ease of notation already now restrict ourselves to two different regimes. The general idea of Markov-switching models, initially introduced by [34], is based on a latent Markov chain that governs the dependence structure of a time series. The later one shall be given in our case by $\left\{\mathbf{U}_{t}=\left(U_{t}^{1}, \ldots, U_{t}^{d}\right)\right\}_{t \in\{1, \ldots, T\}}$ with $\left\{U_{t}^{i}\right\}_{t \in\{1, \ldots, T\}}$ being i.i.d. uniform.

To be precise, let $\left\{S_{t}\right\}_{t=1, \ldots, T}$ be a homogeneous, discrete-time Markov chain, which can take two different states $k=1$ and $k=2$, which we also call regimes. Later on, $k=1$ will represent the "normal" state while $k=2$ will describe the "abnormal" one. The transition matrix shall be denoted by $\mathbf{P}$, having elements given for $k, k^{\prime} \in\{1,2\}$ by $P_{k, k^{\prime}}:=P\left(S_{t}=k^{\prime} \mid S_{t-1}=k\right)$, i.e., the transition probabilities from state $k$ at time $t-1$ to state $k^{\prime}$ at time $t$. Finally, consider two different $R$-vine copula models $\left(\mathcal{V}^{1}, C^{1}, \theta^{1}\right)$ and $\left(\mathcal{V}^{2}, C^{2}, \theta^{2}\right)$ where $\mathcal{V}^{i}, i=\{1,2\}$, are the respective tree structures and $C^{i}$ the corresponding multivariate copulas having parameters stored in the vector $\boldsymbol{\theta}^{i}$.

Our two-regime Markov Switching R-vine (MS-RV) model is therefore given by two $R$-vine copula specifications and the $2 \times 2$ transition matrix $\mathbf{P}$ describing the underlying Markov chain. The conditional copula density is given by

$$
c\left(\mathbf{u}_{t} \mid\left(\mathcal{V}^{i}, C^{i}, \boldsymbol{\theta}^{i}\right)_{i \in\{1,2\}}, S_{t}\right)=\sum_{k=1}^{2} 1_{\{k\}}\left(S_{t}\right) \cdot c\left(\mathbf{u}_{t} \mid\left(\mathcal{V}^{k}, C^{k}, \boldsymbol{\theta}^{k}\right)\right), \quad \mathbf{u}_{t} \in[0,1]^{d} .
$$

Following [38], the full likelihood function of the MS-RV copula model can be decomposed into conditional densities via

$$
\begin{aligned}
f\left(\mathbf{u}_{1}, \ldots \mathbf{u}_{T} \mid \boldsymbol{\theta}^{1}, \boldsymbol{\theta}^{2}, \mathbf{P}\right)= & {\left[\sum_{k=1}^{2} f\left(\mathbf{u}_{1} \mid S_{1}=k, \boldsymbol{\theta}^{k}\right) P\left(S_{1}=k \mid \mathbf{P}\right)\right] } \\
& \times \prod_{t=2}^{T}\left[\sum_{k=1}^{2} f\left(\mathbf{u}_{t} \mid S_{t}=k, \boldsymbol{\theta}^{k}\right) P\left(S_{t}=k \mid \mathbf{u}_{1:(t-1)}, \mathbf{P}\right)\right]
\end{aligned}
$$

where $\mathbf{u}_{1: t}:=\left(\mathbf{u}_{1}, \ldots, \mathbf{u}_{t}\right)$ for $t=\{1, \ldots, T\}$. To tackle the issue of maximizing the above likelihood, Stöber and Czado [38] proposed the following stepwise Expectation Maximization (EM) algorithm based on the procedure of [61]:

1. Given the current parameters $\left(\boldsymbol{\theta}^{1, l}, \boldsymbol{\theta}^{2, l}, \mathbf{P}^{l}\right)$ calculate iteratively the so-called "smoothed" probabilities

$$
\left(\Omega_{t \mid T}\left(\left(\boldsymbol{\theta}^{1, l}, \boldsymbol{\theta}^{2, l}, \mathbf{P}^{l}\right)\right)_{s_{t}}:=P\left(S_{t}=s_{t} \mid \mathbf{u}_{1: T},\left(\boldsymbol{\theta}^{1, l}, \boldsymbol{\theta}^{2, l}, \mathbf{P}^{l}\right)\right), \quad s_{t} \in\{1,2\},\right.
$$

via a Hamilton filter (cf. [34,38] for details).

2. Maximize the pseudo log-likelihood function

$$
\begin{gathered}
Q\left(\left(\boldsymbol{\theta}^{1, l+1}, \boldsymbol{\theta}^{2, l+1}, \mathbf{P}^{l+1}\right) ; \mathbf{u}_{1: T},\left(\boldsymbol{\theta}^{1, l}, \boldsymbol{\theta}^{2, l}, \mathbf{P}^{l}\right)\right) \\
:=\sum_{s_{1}=1}^{2} \ldots \sum_{s_{T}=1}^{2} \log \left(f\left(\mathbf{u}_{1: T}, s_{1: T} \mid\left(\boldsymbol{\theta}^{1, l+1}, \boldsymbol{\theta}^{2, l+1}, \mathbf{P}^{l+1}\right)\right)\right) P\left(s_{1: T}=s_{1: T} \mid \mathbf{u}_{1: T},\left(\boldsymbol{\theta}^{1, l}, \boldsymbol{\theta}^{2, l}, \mathbf{P}^{l}\right)\right) \\
\propto \quad \sum_{t=1}^{T}\left[\sum_{s_{1}=1}^{2} \ldots \sum_{s_{T}=1}^{2} \log \left(f\left(\mathbf{u}_{t,} \mid S_{t}=s_{t},\left(\boldsymbol{\theta}^{1, l+1}, \boldsymbol{\theta}^{2, l+1}\right)\right)\right) P\left(S_{1: T}=s_{1: T} \mid \mathbf{u}_{1: T},\left(\boldsymbol{\theta}^{1, l}, \boldsymbol{\theta}^{2, l}, \mathbf{P}^{l}\right)\right)\right] \\
+\sum_{s_{1}=1}^{2} \ldots \sum_{s_{T}=1}^{2}\left[\sum_{t=1}^{T} \log \left(P\left(S_{t}=s_{t} \mid S_{t-1}, \mathbf{P}^{l+1}\right)\right)+\log \left(P\left(S_{1}=s_{1}\right)^{l+1}\right)\right] \\
\times P\left(S_{1: T}=s_{1: T} \mid \mathbf{u}_{1: T},\left(\boldsymbol{\theta}^{1, l}, \boldsymbol{\theta}^{2, l}, \mathbf{P}^{l}\right)\right)
\end{gathered}
$$


stepwise, starting with respect to $\left(\boldsymbol{\theta}^{1, l+1}, \boldsymbol{\theta}^{2, l+1}\right)$ which is carried out sequentially over the vine trees. Afterwards, the maximization with respect to $\mathbf{P}^{l+1}$ can be done analytically via the formula of [69].

We want so stress that the above is not the proper EM algorithm and therefore known convergence results (cf. [70,71]) do not hold. However, as the authors of [38] pointed out, the classical EM procedure does rely on the correct maximization for each step which is impossible to ensure in our case as we have to invoke numerical methods. Nevertheless, tree-wise estimation of $R$-vine structures is asymptotically consistent and therefore the stepwise EM algorithm is still a close approximation to the classical EM algorithm. For more details we refer the interested reader to [38].

In the light of the above and to evaluate our estimation results further, we shall carry out a simulation study to obtain empirical confidence bounds for $\lambda_{Q T D}$ within our Markov switching setup in the next section.

\section{Determining "Normal" and "Abnormal" Regimes and Suitable $R$-Vine Structures}

The previous section outlined our MS-RV model setup which requires pre-defined $R$-vine structures for both regimes as input factors. Therefore, starting with the trees from model (2-dependent), we need to identify potential changes within the dependence structure of our index data over time. In order to do that, we shall follow [38] and perform a detailed rolling window analysis (RWA) for the bivariate copulas within and between continents. Based on each pair's QTD over time, we will motivate a classification of what is "normal" and "abnormal" to specify our regimes. Afterwards, making use of these suitable pre-defined $R$-vine structures, we start by estimating the MS-RV setup for each region separately, resulting in a global model assuming independent continents which we shall call (3-independent-MS). Finally, (3-dependent-MS) will be the corresponding Markov-switching setting for (2-dependent) which, up until now, performed best.

\subsection{Rolling Window Analysis}

For the following procedure, we choose 250-day windows into the future and consider which of our copulas out of Gauss, Student- $t$ and all Gumbel rotations fits best. Additionally, the value of Kendall's $\tau$ and the QTD from Definition 4 is calculated as well. Therefore, in particular, we will be able to identify times of high and low tail dependence. As we are only considering bivariate pairs for now, no $R$-vine models are needed.

The outcome of the RWA is structured graphically as follows: For each index pair, the first graph shows the fitted copula family: Gauss (Ga), Student- $t$ (St-t), Gumbel (Gu), Gumbel90 (Gu90), Gumbel180 (Gu180) and Gumbel270 (Gu270). The second graph depicts the series of Kendall's $\tau$ based on (5) and as a horizontal line it's empirical value over the whole observation period. Finally, the third graph illustrates the QTDs and their mean over all calculated values. The results for the first trees can be found in Figures 8-11. These show that there are strong indications for regime shifts within the bivariate relationships of most index pairs. However, the plots for all higher order trees would not paint such a clear picture. In fact, the chosen copula families tend to vary more which can be explained by our earlier observations from Section 4 that the values of Kendall's $\tau$ for the second and third trees are generally much lower as for the first ones.

Considering the depicted results in more detail, we realize that especially for the respective equity-volatility pairs, Gumbel90 seems to be the standard regime indicating a nearly constant asymmetric tail dependence which is further supported by the QTD values. In the case of an equity-commodity relation, i.e., SPX-BBC, Kendall's $\tau$ as well as the QTD values increased rather sharply in 2008 and started to came back to their previous levels near zero 2014. This clearly illustrates the vanishing diversification in a global crisis, as the strength of positive dependence between equity and commodities tends to increase during bad market times. 
Copula Family
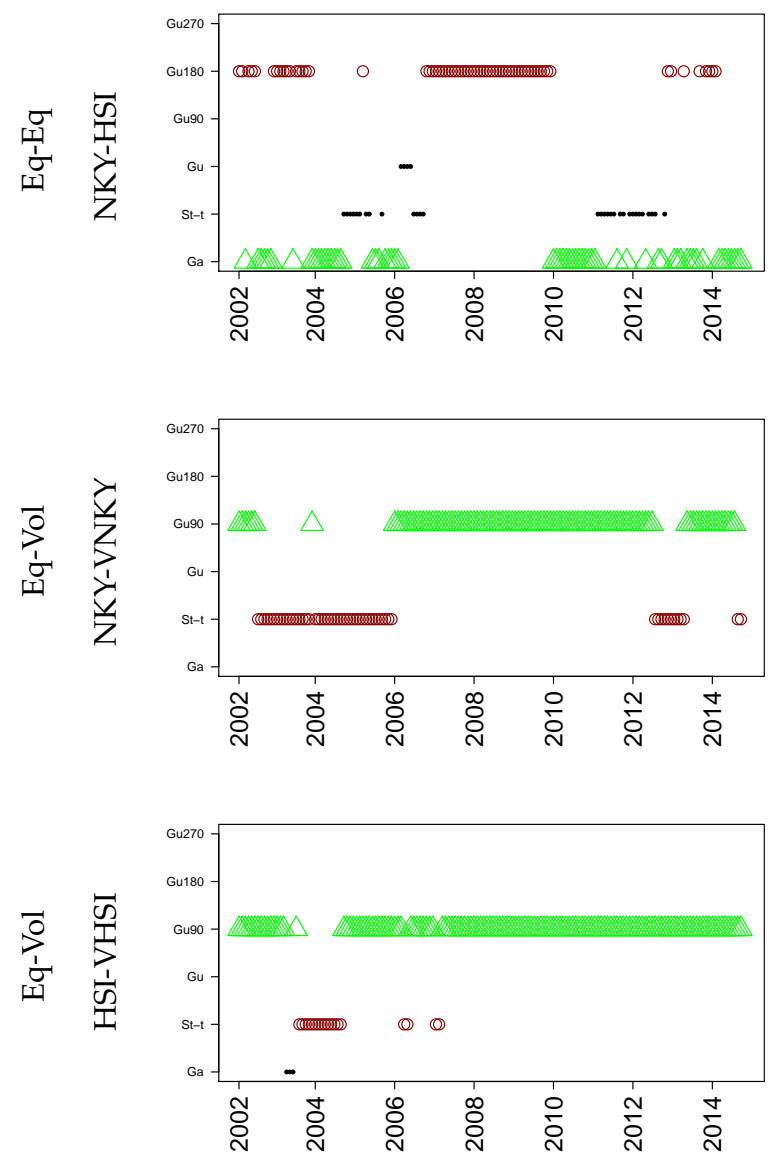

Kendall's Tau
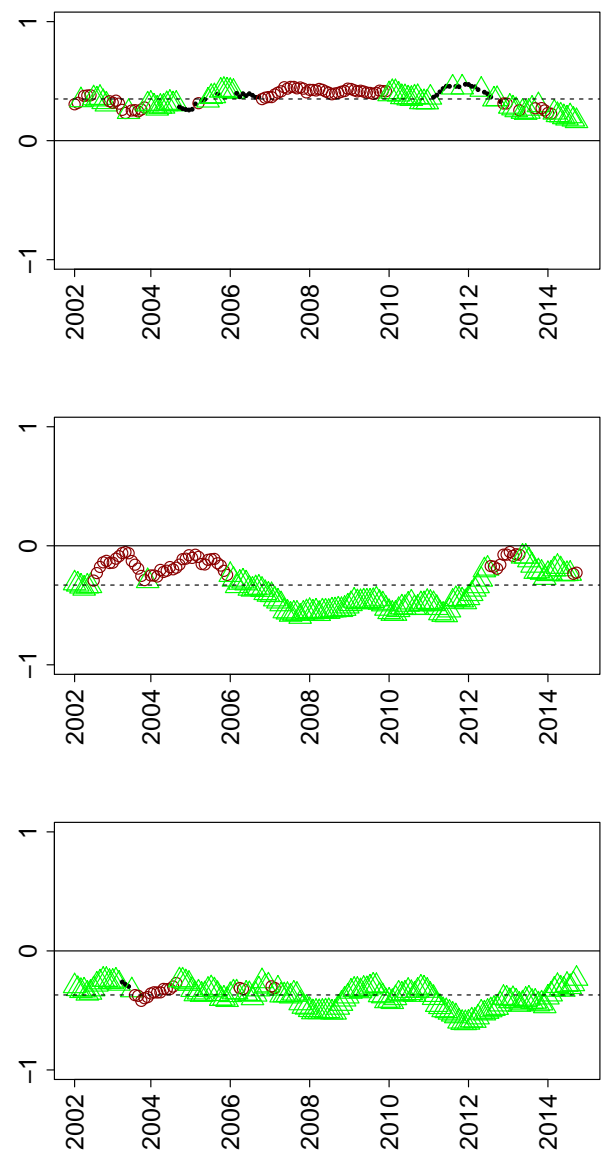

Quarter Tail Dependence
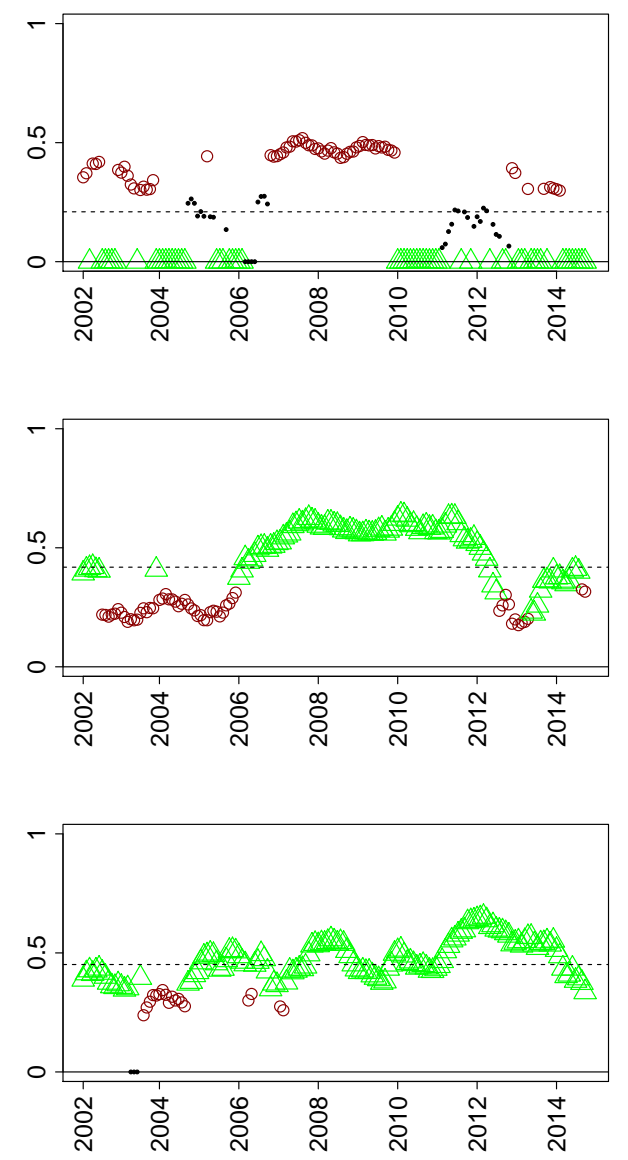

Figure 8. Asia-250 day rolling window analysis for bivariate copulas in the first tree. Periods are marked in green/triangle and red/star, respectively, when copula families chosen to represent "normal" and "abnormal" regimes were fitted. For reasons of visibility, only every 20th data point is plotted. 
Copula Family
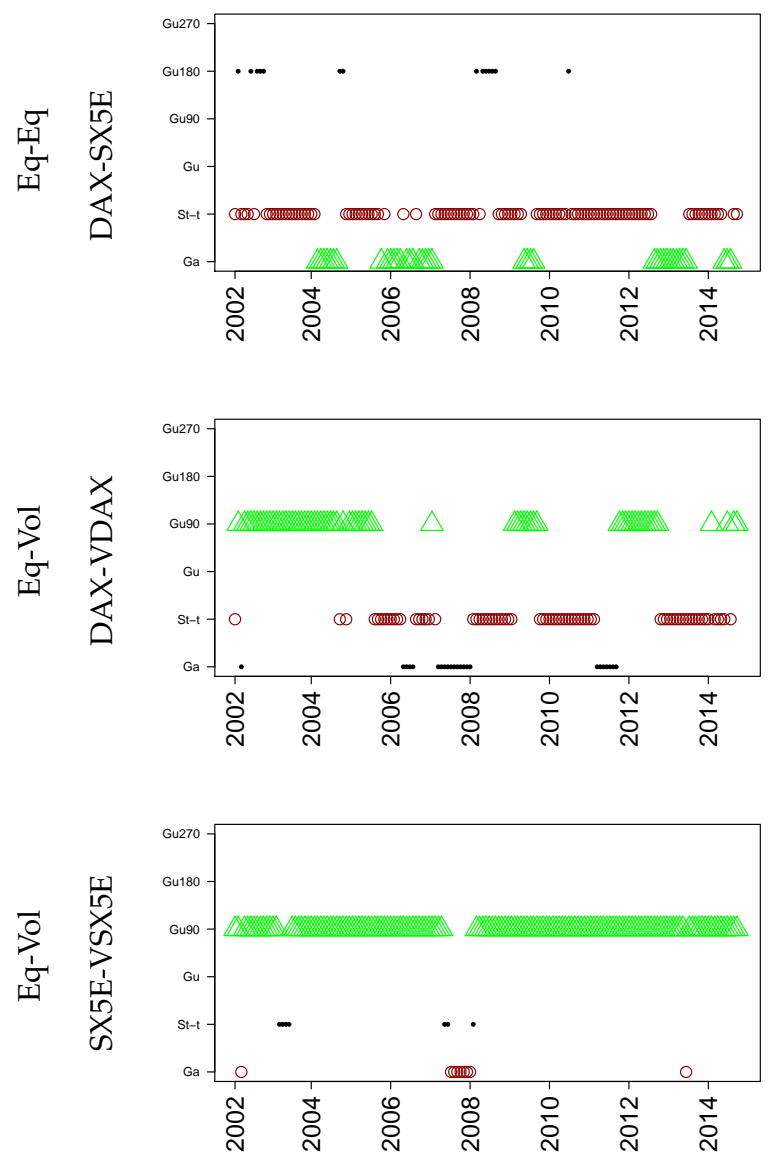

Kendall's Tau
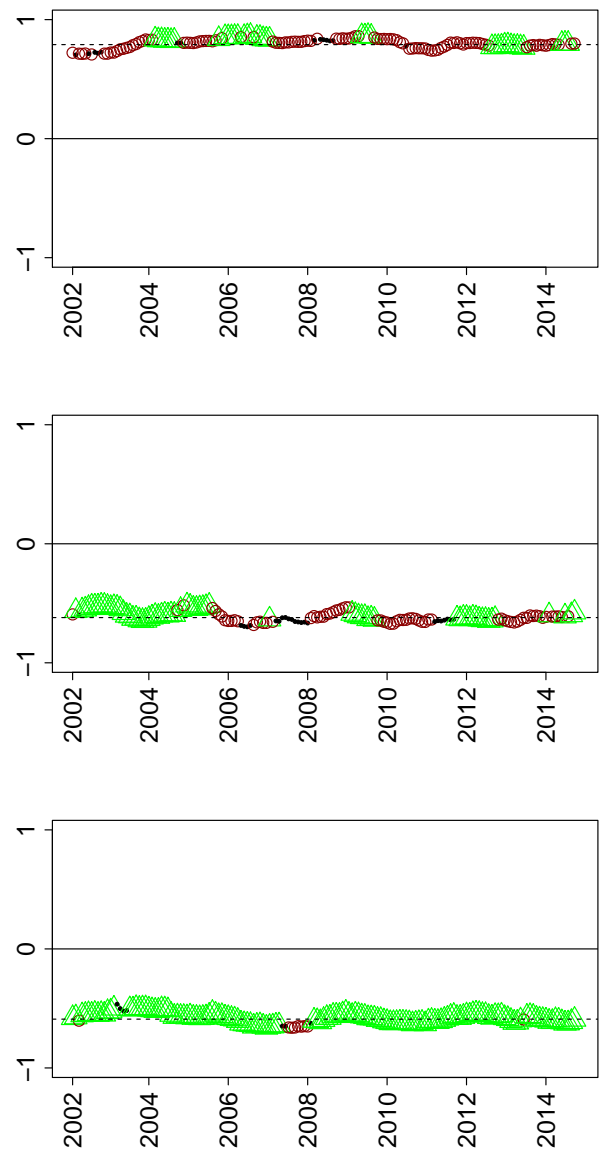

Quarter Tail Dependence
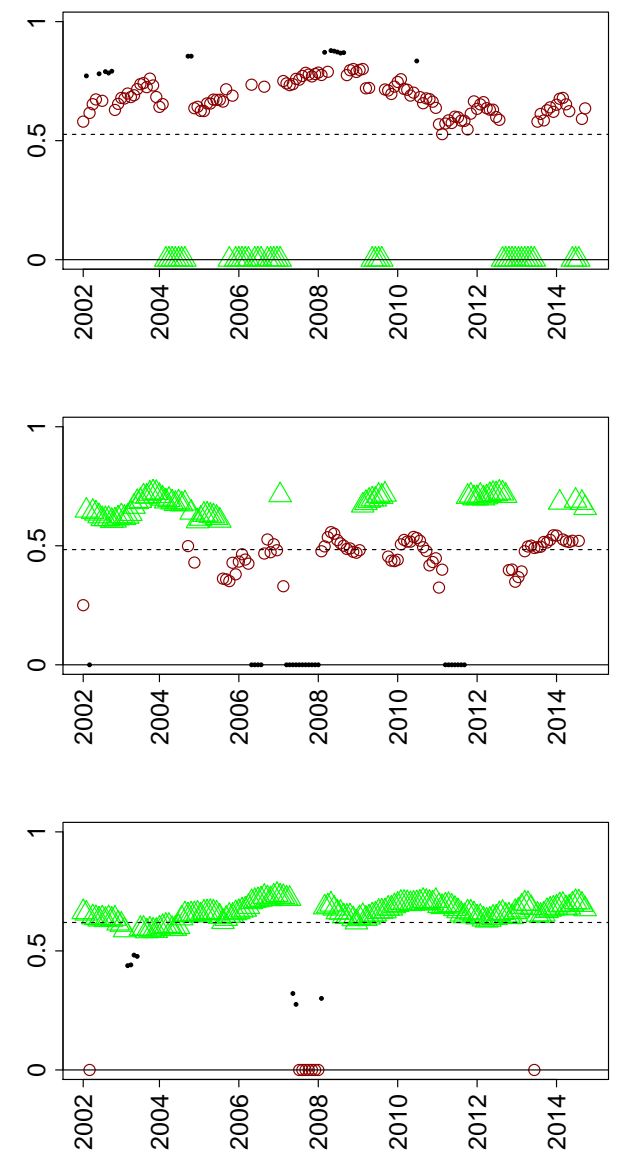

Figure 9. Europe-250 day rolling window analysis for bivariate copulas in the first tree. Periods are marked in green/triangle and red/star, respectively, when copula families chosen to represent "normal" and "abnormal" regimes were fitted. For reasons of visibility, only every 20th data point is plotted. 
Copula Family
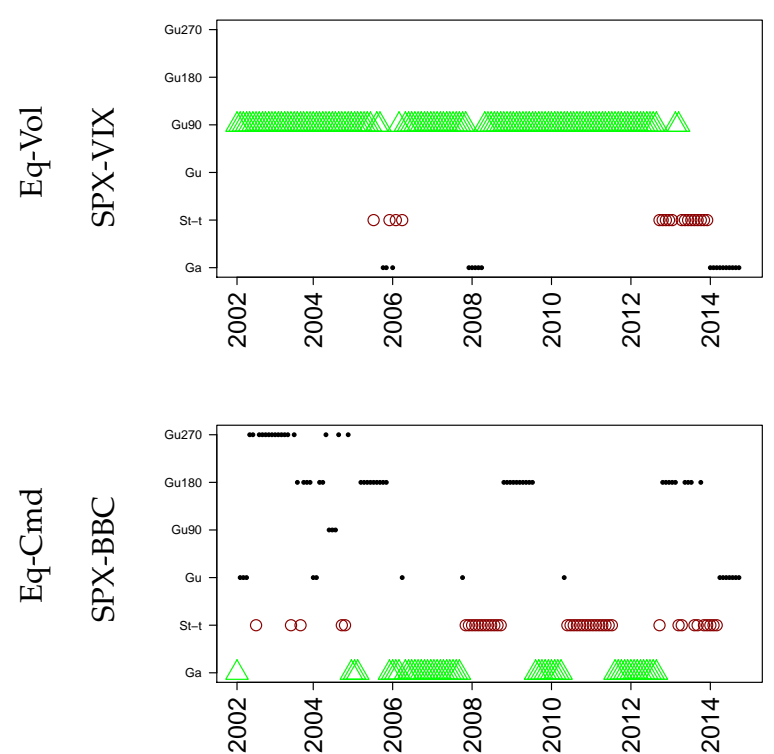

Kendall's Tau
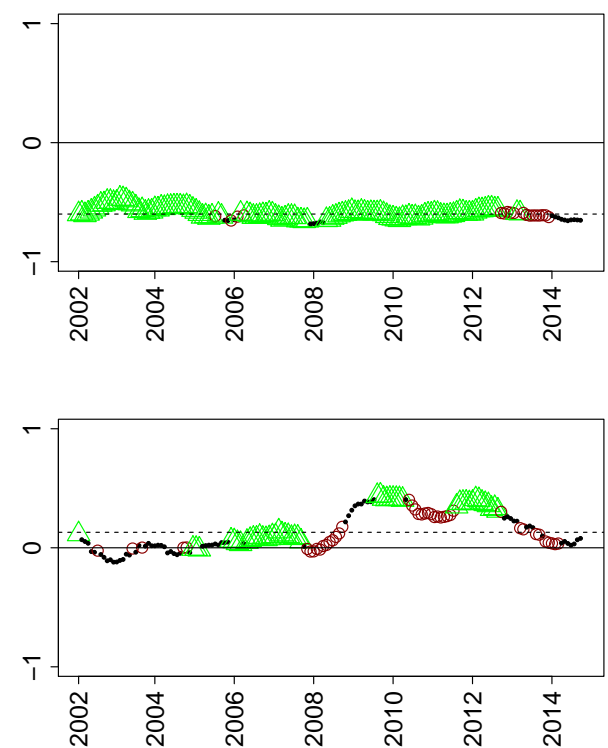

Quarter Tail Dependence
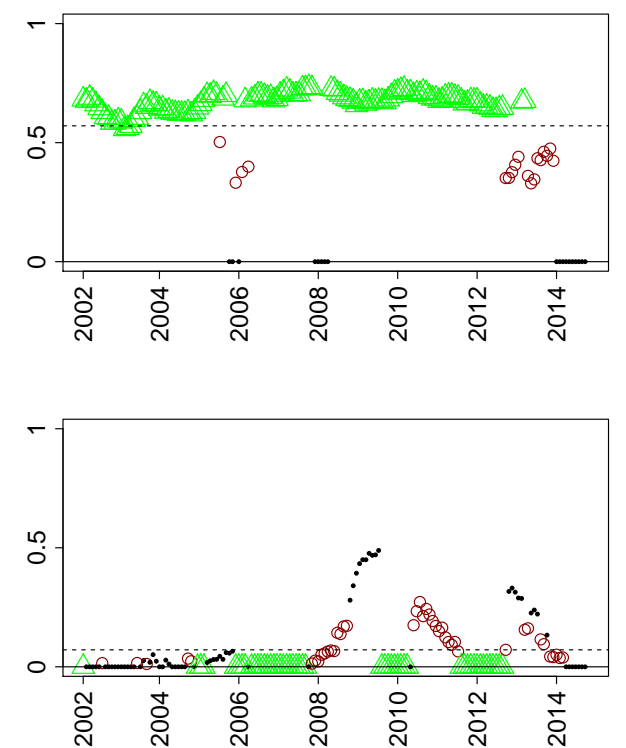

Figure 10. USA-250 day rolling window analysis for bivariate copulas in the first tree. Periods are marked in green/triangle and red/star, respectively, when copula families chosen to represent "normal" and "abnormal" regimes were fitted. For reasons of visibility, only every 20th data point is plotted. 
Copula Family
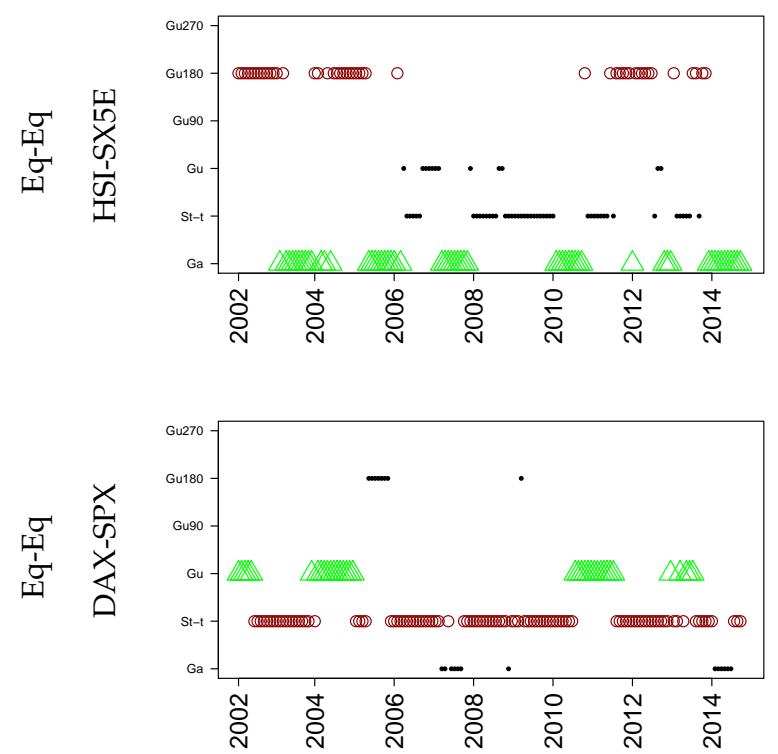

Kendall's Tau
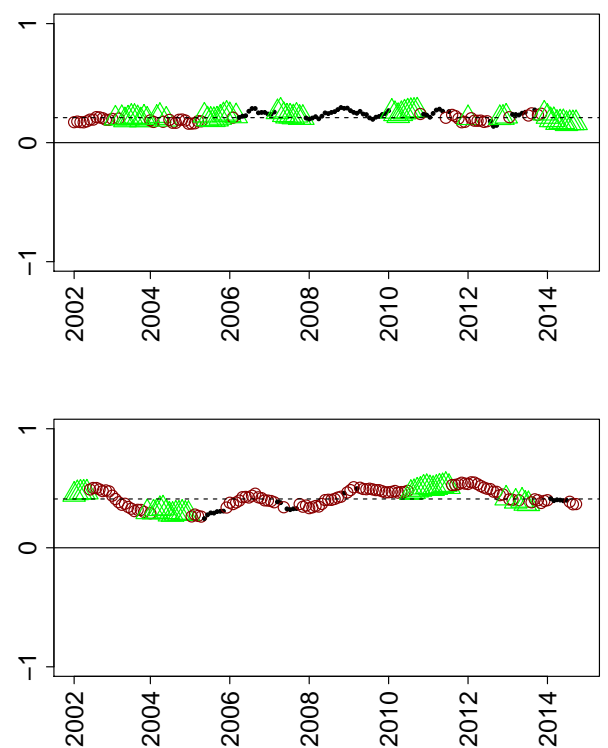

Quarter Tail Dependence
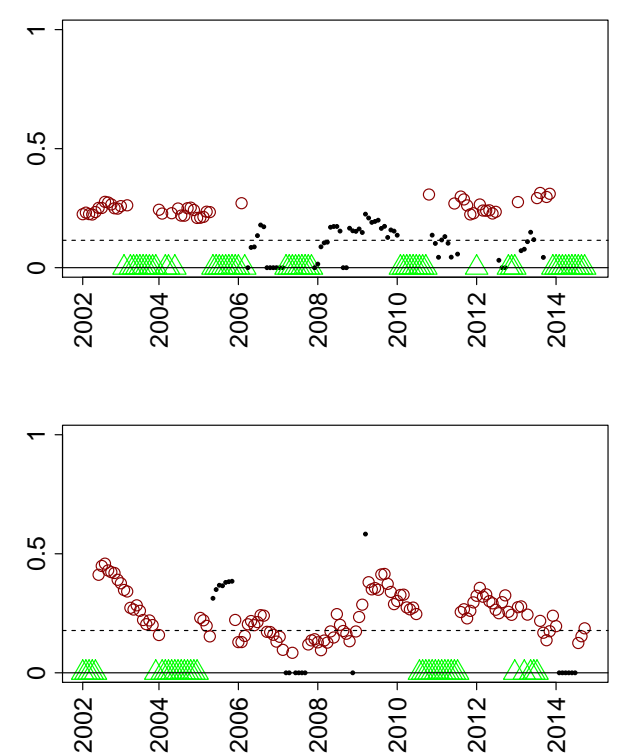

Figure 11. Between continents-250 day rolling window analysis for bivariate copulas in the first tree. Periods are marked in green/triangle and red/star, respectively, when copula families chosen to represent "normal" and "abnormal" regimes were fitted. For reasons of visibility, only every 20th data point is plotted. 
The equity-equity index pairs on the flipside do not show such a clear picture: While Kendall's $\tau$ is comparably high for DAX-SX5E and DAX-SPX and the Gauss/Student- $t$ copulas are predominantly chosen, the connection between the developed regions and China given by HSI-SX5E as well as NKY-HSI seems weaker and tends to either a Gaussian or Gumbel180 copula. However, the QTD values are generally higher in abnormal times like 2007-2010 or 2010-2012-the only exception being DAX-SX5E which shows quite a strong QTD for nearly the whole period. Anyhow, this can be explained by the fact that the DAX is partly included within the SX5E.

Having considered the RWA results as a kind of explanatory analysis, we want to motivate and specify how "normal" and "abnormal" shall be classified within our Markov-switching setup, i.e., what kind of behavior in the dependence structure of our indices is usually not expected in "normal" times. For potential regime switches within the first trees, we propose the algorithm depicted by Figure 12: Firstly, the two copula families that appear most often within the RWA are identified for each index pair; Secondly, we differentiate between pairs with overall positive and negative dependence measured by the empirical Kendall's $\tau$. In case of positive dependence, the "abnormal" regime should be the one with the higher QTD among the two, which is in line with our findings above. For negative values of the empirical Kendall's $\tau$, as can be found for, e.g., equity-volatility pairs, the "abnormal" state is identified as the one with the lower QTD corresponding to the fact that Gumbel90 has been shown to be the "normal" regime. For all trees of higher orders, justified by the rather low values of Kendall's $\tau$, we shall fix the copula family with the highest frequency in the RWA and allow only the individual parameters to switch between regimes. The final classifications are summarized by Table 8 . We are aware that recent work on market integration like [72,73] shows that equity tail dependencies have increased over the last decades which would imply a strengthening tendency towards the "abnormal" regime. However, our considered time frame only starts in 2002 and the RWA does not indicate any such trends in our (shorter) time period. Additionally, our model also includes volatility indices where a potentially increasing QTD would prefer the "normal" state again.

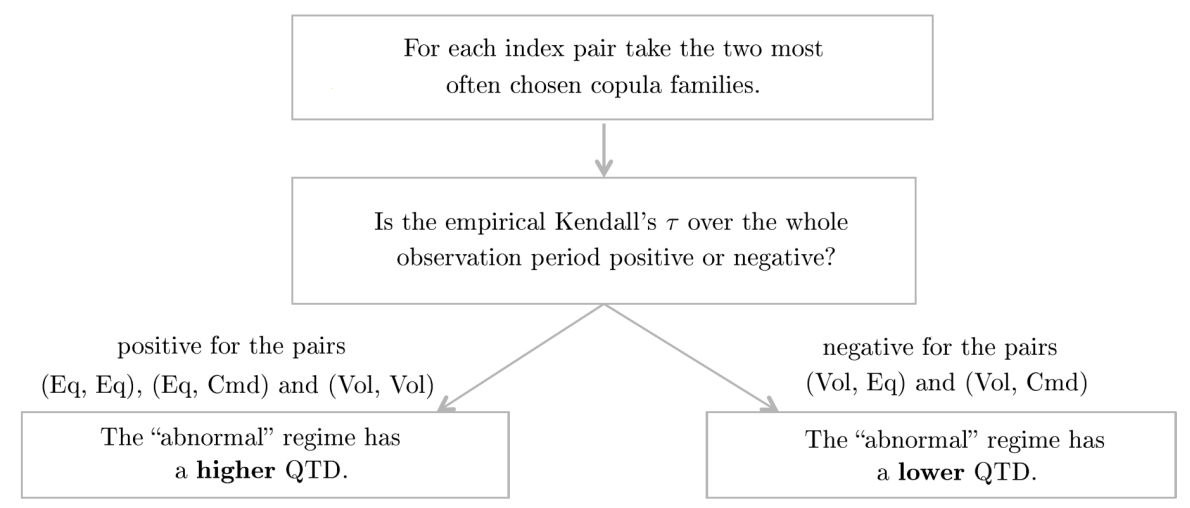

Figure 12. Classification algorithm for determining "normal" and "abnormal" regimes within the first trees based on Kendall's $\tau$ and quarter tail dependence (QTD).

As in [38], before running the EM algorithm from Section 5 to estimate the MS-RV model, we want to check if our chosen regimes are sensible by considering the differences in the log-likelihoods over time. For that, both copula families for the "normal" and "abnormal" regimes in the first tree are fitted separately using again 250-day rolling windows. Figure 13 summarizes the results by plotting the difference

$$
\log L\left(C_{\text {abnormal }}\right)-\log L\left(C_{\text {normal }}\right),
$$

i.e., positive values indicate an outperformance of the "abnormal" regime. Additionally, the periods are marked, when the copula families identified with "normal" and "abnormal" regimes were fitted in the RWA. The plotted results confirm that our algorithm (cf. Figure 12) seems a sensible choice. 
Table 8. Results of our algorithm (Figure 12): "Normal" and "abnormal" regime classification by frequency and quarter tail dependence $\lambda_{Q T D}$ for the first trees. For all higher trees the copulas with the highest frequencies in the RWA are chosen and only the individual parameters are allowed to switch.

\begin{tabular}{|c|c|c|c|c|c|c|c|c|}
\hline & \multirow{2}{*}{ Index Pair } & \multirow{2}{*}{ Kendall's $\tau$} & \multicolumn{3}{|c|}{ “Normal" Regime } & \multicolumn{3}{|c|}{ "Abnormal" Regime } \\
\hline & & & Family & Frequency & $\lambda_{Q T D}$ & Family & Frequency & $\lambda_{Q T D}$ \\
\hline \multicolumn{9}{|c|}{ Asia } \\
\hline Eq-Eq & NKY-HSI & $>0$ & Gauss & 0.39 & 0 & Gumbel180 & 0.41 & 0.43 \\
\hline Eq-Vol & NKY-VNKY & $<0$ & Gumbel90 & 0.67 & 0.51 & Student- $t$ & 0.33 & 0.24 \\
\hline Eq-Vol & HSI-VHSI & $<0$ & Gumbel90 & 0.88 & 0.48 & Student- $t$ & 0.11 & 0.30 \\
\hline Eq-Vol & HSI-VNKY|NKY & $<0$ & Gumbel90 & 0.48 & 0.10 & Gumbel90 & 0.48 & 0.10 \\
\hline Eq-Vol & NKY-VHSI|HSI & $<0$ & Gauss & 0.37 & 0 & Gauss & 0.37 & 0 \\
\hline Vol-Vol & VNKY-VHSI|NKY, HSI & $>0$ & Gauss & 0.49 & 0 & Gauss & 0.49 & 0 \\
\hline \multicolumn{9}{|c|}{ Europe } \\
\hline $\mathrm{Eq}-\mathrm{Eq}$ & DAX-SX5E & $>0$ & Gauss & 0.24 & 0 & Student- $t$ & 0.69 & 0.68 \\
\hline Eq-Vol & DAX-VDAX & $<0$ & Gumbel90 & 0.42 & 0.67 & Student- $t$ & 0.43 & 0.47 \\
\hline Eq-Vol & SX5E-VSX5E & $<0$ & Gumbel90 & 0.91 & 0.66 & Gauss & 0.04 & 0 \\
\hline Eq-Vol & SX5E-VDAX|DAX & $<0$ & Gauss & 0.40 & 0 & Gauss & 0.40 & 0 \\
\hline Eq-Vol & DAX-VSX5E|SX5E & $<0$ & Gumbel270 & 0.31 & 0 & Gumbel270 & 0.31 & 0 \\
\hline Vol-Vol & VDAX-VSX5E|DAX,SX5E & $>0$ & Student- $t$ & 0.66 & 0.33 & Student- $t$ & 0.66 & 0.33 \\
\hline \multicolumn{9}{|c|}{ USA } \\
\hline Eq-Vol & SPX-VIX & $<0$ & Gumbel90 & 0.78 & 0.67 & Student- $t$ & 0.12 & 0.40 \\
\hline $\mathrm{Eq}-\mathrm{Cmd}$ & SPX-BBC & $>0$ & Gauss & 0.32 & 0 & Student- $t$ & 0.25 & 0.10 \\
\hline Vol-Cmd & VIX-BBC|SPX & $<0$ & Gauss & 0.38 & 0 & Gauss & 0.38 & 0 \\
\hline \multicolumn{9}{|c|}{ Between continents } \\
\hline Eq-Eq & HSI-SX5E & $>0$ & Gauss & 0.34 & 0 & Gumbel180 & 0.32 & 0.25 \\
\hline Eq-Eq & DAX-SPX & $>0$ & Gumbel & 0.22 & 0 & Student- $t$ & 0.62 & 0.25 \\
\hline
\end{tabular}




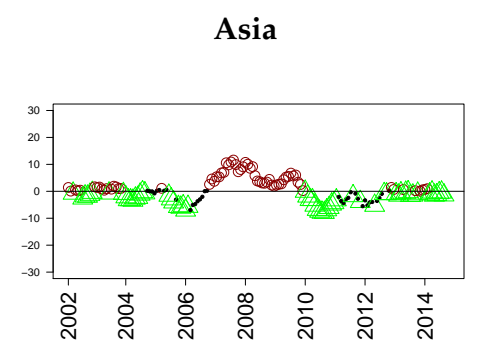

(a) NKY-HSI

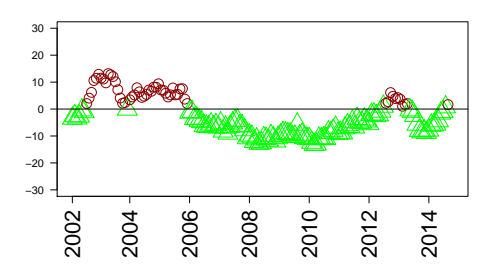

(e) NKY-VNKY

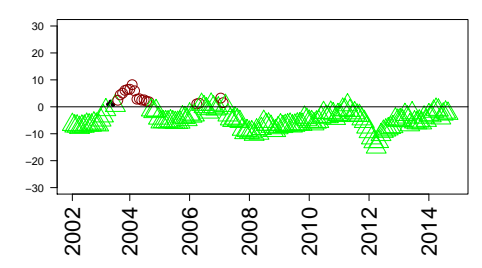

(i) HSI-VHSI
Europe

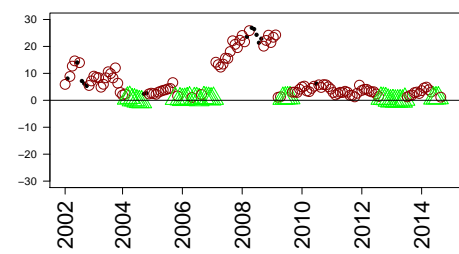

(b) DAX-SX5E

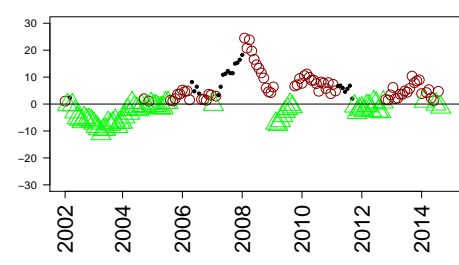

(f) DAX-VDAX

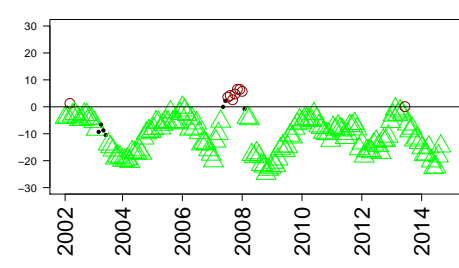

(j) SX5E-VSX5E
North-America

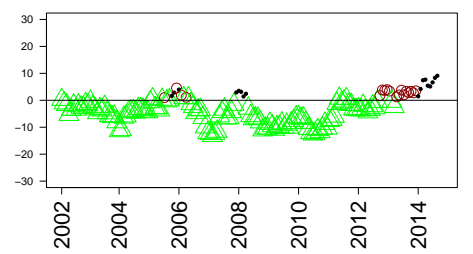

(c) SPX-VIX

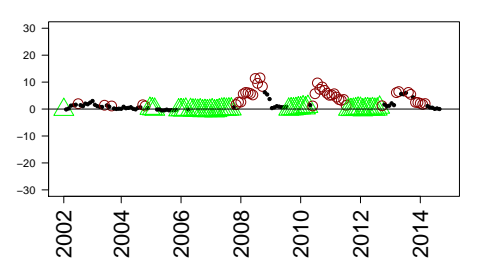

(g) SPX-BBC
Between continents

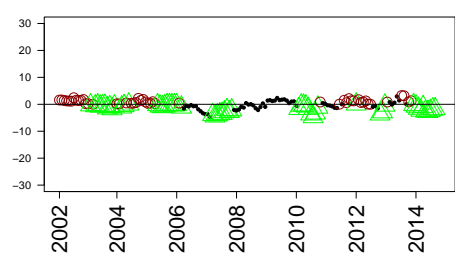

(d) HSI-SX5E

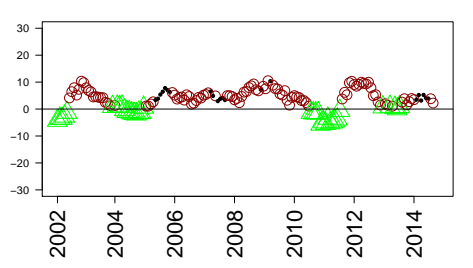

(h) DAX-SPX

Figure 13. Difference in the log-likelihoods between the fit of the copula family chosen to represent "abnormal" and "normal" regime. Positive values indicate outperformance of the "abnormal" regime. Again, periods are marked as in Figures 8-11. 


\subsection{Results on MS-RV Models}

We start by considering each region individually again for which we use the following pre-defined $R$-vine structures: The first trees were taken as determined in Table 8 based on the algorithm from Figure 12, i.e., we have generally different copula setups for each regime, while all higher trees are chosen in line with (2-independent) and the algorithm, letting only the copula parameters switch. For more details on the exact $R$-vine structures and estimated parameters we refer to the Appendix A.

For all three continents, compared with their static counterparts, the MS-RV model performs better in terms of log-likelihood, AIC and BIC as outlined by Table 9. The probabilities of being in the "abnormal" regime, smoothed by a 10-day moving average, can be seen in the first three plots of Figure 14 .

Table 9. Log-likelihood, AIC and BIC for the static R-vine models Asia 1 , Europe ${ }_{1}, \mathrm{USA}_{1}$ and their Markov-switching counterparts.

\begin{tabular}{cccc}
\hline & Log-Likelihood & AIC & BIC \\
\hline Model Asia $_{1}$ & 2027.61 & -4041.22 & -3998.24 \\
Model Asia $_{1, \text { MS }}$ & $\mathbf{2 1 1 8 . 5 9}$ & $-\mathbf{4 2 0 5 . 1 8}$ & $-\mathbf{4 1 0 6 . 9 2}$ \\
\hline Model Europe $_{1}$ & 8973.48 & $-17,924.95$ & $-17,857.40$ \\
Model Europe $_{1, \mathbf{M S}}$ & $\mathbf{9 0 5 3 . 7 2}$ & $\mathbf{- 1 8 , 0 7 1 . 4 4}$ & $\mathbf{- 1 7 , 9 6 0 . 8 9}$ \\
\hline Model USA $_{1}$ & 2017.63 & -4027.26 & -4002.69 \\
Model USA $_{1, \text { MS }}$ & $\mathbf{2 0 7 4 . 6 0}$ & $-\mathbf{4 1 3 5 . 2 0}$ & $-\mathbf{4 0 9 2 . 2 2}$ \\
\hline
\end{tabular}
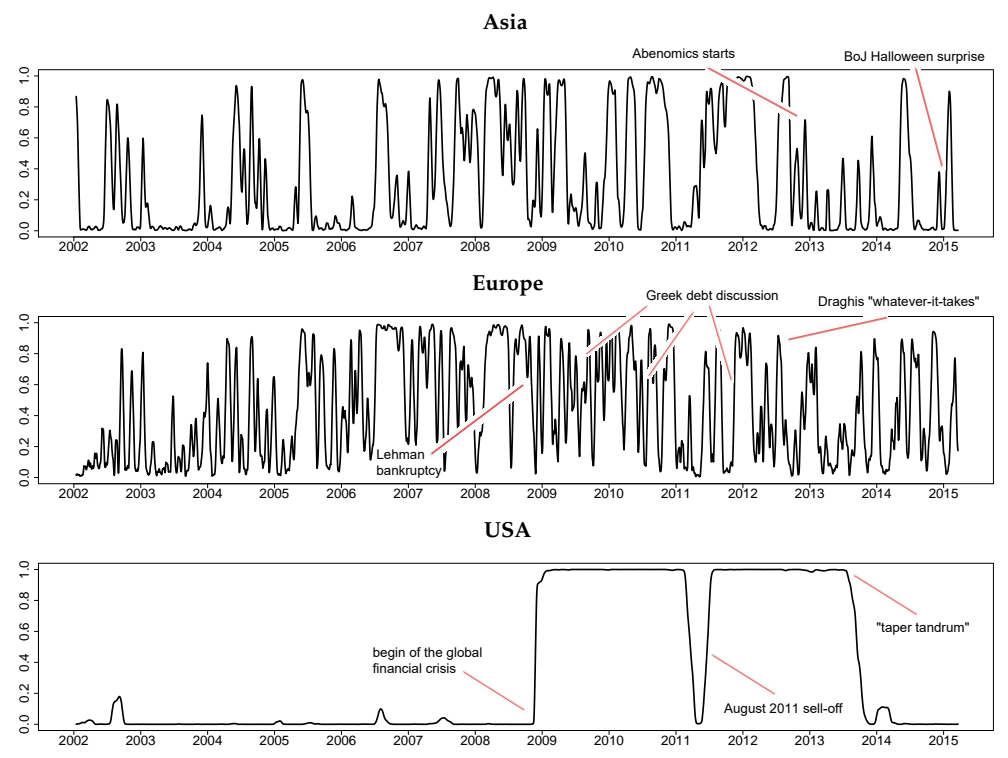

(Corrected) Pearson contingency coefficient

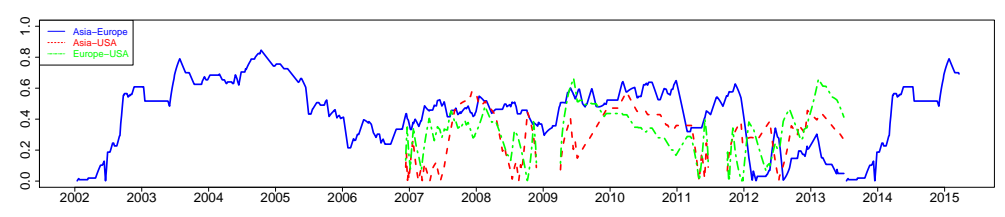

Joint Model

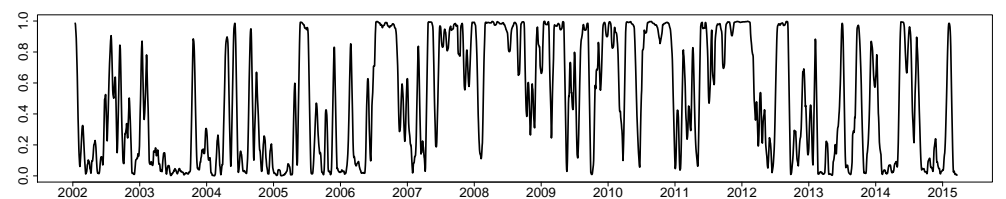

Figure 14. Probabilities of being in the "abnormal" regime smoothed by 10-day moving average for model (3-independent-MS) and (3-dependent-MS). The (corrected) Pearson contingency coefficients have been calculated over a 500-day rolling window into the future. 
In the US we see a strong predominance of the "normal" regime pre-Lehman while after the start of the global financial crisis and up until the summer of 2013 the "abnormal" regime prevails. Even though the probability plot looks very much different to those from Asia or Europe, there is a neat explanation: Having the results of the RWA in mind (cf. Figure 10, especially the movement of the QTD), the regime switch to "abnormal" seems to be mainly driven by SPX-BBC as the general dependence structure of SPX-VIX is rather stable over time. This fits neatly to well-known results stating that the tail dependence (and therefore the QTD) increases between equities (and commodities) in times of financial stress (cf. [28], for example). In fact, when we only consider a bivariate switching model for SPX-VIX, the probability plot would look like those for Asia or Europe-although the difference between the MS-RV model and the static ones (using the fixed regime structures) is not that pronounced in terms of the log-likelihood. Doing the same for SPX-BBC, we get a graph much similar to the very smooth US plot with distinct regimes and a very low switching probability. Also, the advantage of the MS-RV model compared to the static ones is notably larger for SPX-BBC than for SPX-VIX (in line with Figure 10). Therefore, the equity-commodity relationship mainly dominates the common US switching model.

In June 2013, the Federal Reserve announced the potential scaling back of its quantitative easing programm later that year leading to a more than $4 \%$ fall in the equity markets and a spike in volatility ("taper tandrum"). Interestingly, this regime change can not be seen directly in the first tree and is merely driven by the parameter switches in the higher trees. The drop in the probability in early 2011 goes hand-in-hand with the fall in the QTD of SPX-BBC while the rise in mid-2011 can be explained by the equity market sell-off and spike in implied volatility in August 2011 which is again better reflected in the higher order trees.

Compared to the US, the plots for Asia and Europe clearly show a greater activity in terms of regime switching which mostly seems to happen at the same time, indicating that a joint MS-RV model could be a sensible choice. To further investigate this hypothesis, we employ the following, heuristic procedure: For each continent, if the probability of being in the "abnormal" regime is smaller or equal $50 \%$ we assume that the respective region is currently in the "normal" state and vice versa. For the resulting categorical time series, we calculate the (corrected) Pearson contingency coefficients based on a 500-day rolling window into the future. The results are depicted in the fourth plot of Figure 14. If for some windows, at least one series does not indicate a switching and is therefore constant, the relevant window is dropped from the plot. The picture is somewhat ambiguous, however Asia and Europe clearly hint that a joint switching model might be worth investigating.

We want to stress again that by definition of our "abnormal" regimes, these do not necessarily indicate a crisis in financial markets. For example, the Halloween surprise by the Bank of Japan on 31 October 2014 when Governor Haruhiko Kuroda unexpectedly increased his QQE program by an annual pace of 30 trillion JPY is obviously not a crisis but can still be matched with a rising probability of being in the "abnormal" regime. A similar feature can be seen in Japan throughout the years 2013: Due to the election of the Liberal Democrats under the leadership of Shinzo Abe in December 2012 and the subsequent implementation of "Abenomics", both, the equity markets and implied volatility started to rise indicating an "abnormal" regime (which can also be seen in the RWA). However in Hong Kong, the HSI had a rather bad year (cf. Figure 1) with a rising VHSI drawing the probability plot down to the "normal" state again and again, a behavior which is in line with the known asymmetric dependencies between equity and volatility (cf. $[9,74])$. 
On the other side, the rather high frequency of switches in Europe especially in 2009-2012 can be explained by the ongoing Greek debt discussion (i.e., a classical crisis) ending with Mario Draghis famous "whatever it takes"-speech on 26 July 2012. What might be surprising on a first view is that the Lehman crisis brought the probability plot down and close to zero in the subsequent months. However, this can again be explained by the "normal" regime's definition and a look at the RWA: While for the equity markets, the "abnormal" regime might be sensible, the historically large rise in implied volatility in both, the VDAX and the VSX5E (cf. Figure 1), occurring at the same time draws our model back to the "normal" state which means a Gumbel90 copula for DAX-VDAX and SX5E-VSX5E.

Assuming independent continents and adding the log-likelihoods up, unsurprisingly shows that the above setup, called (3-independent-MS) outperforms its benchmark (2-independent), cf. Table 10. However, as we noted especially for Asia and Europe, switches seem to occur mostly at the same time, even though the special behavior of the USA would not directly support that hypothesis. Therefore as a final investigation, we estimate a joint MS-RV model, implying regime changes at the same time, which we shall call (3-dependent-MS). Again, the first trees are based on Table 8 while the higher order dependencies (tree structure and copula families) were chosen in line with (2-dependent) allowing only the copula parameters to switch. In terms of log-likelihood and AIC, the MS-RV model is superior to the static setup while BIC (which penalizes the amount of parameters more than AIC) rather supports the later one, cf. Table 10. The smoothed probability plot of model (3-dependent-MS) can be found at the bottom of Figure 14. Most of the previously identified periods of the "abnormal" regime appear here as well.

Table 10. Log-likelihood, AIC and BIC for the global, static $R$-vine models (2-independent) and (2-dependent) compared to their Markov-switching counterparts (3-independent-MS) and (3-dependent-MS).

\begin{tabular}{cccc}
\hline & Log-Likelihood & AIC & BIC \\
\hline (2-independent) & $13,018.72$ & $-25,993.44$ & $-25,858.33$ \\
(3-independent-MS) & $\mathbf{1 3 , 2 4 6 . 9 1}$ & $-\mathbf{2 6 , 4 1 1 . 8 1}$ & $-\mathbf{2 6 , 1 6 0 . 0 2}$ \\
\hline (2-dependent) & $14,618.70$ & $-29,091.40$ & $-\mathbf{2 8 , 6 4 3 . 0 9}$ \\
(3-dependent-MS) & $\mathbf{1 4 , 7 3 8 . 8 8}$ & $-\mathbf{2 9 , 1 9 1 . 7 6}$ & $-28,313.56$ \\
\hline
\end{tabular}

As discussed at the end of the previous section, known convergence results for the proper EM algorithm do not hold in our case. Therefore we shall invoke a simulation study to stress the reliability of our applied estimation technique: In order to do that, we simulate 1000 multivariate sample 'paths', each having a total length of 1000 copula returns from (3-dependent-MS) initially starting in the "normal" regime and re-estimate the joint MS-RV model via the method outlined in the previous section. For better visibility we shall focus on the QTDs of the first trees and present the initially estimated values compared to the means and empirical confidence bounds obtained by our simulation study. Additionally, we carry out the same procedure for the switching probabilities in each regime. The results are summarized in Table 11: All obtained QTD-values can be recovered quite well even though no formal theory is available. Also, for all pairs the confidence bounds for the "normal" and "abnormal" regimes do not overlap, which confirms that we actually identified two distinct regimes in our global data set. 
Table 11. $\lambda_{Q T D}$ and switching probabilities for (3-dependent-MS) with mean and 5\%-confidence intervals obtained by bootstrapping: Using the initially estimated parameters and starting in the "normal" regime we simulated 1000 times 1000 copula-data points and calculated $\lambda_{Q T D}$ by re-estimating (3-dependent-MS), considering the respective parameters in the first trees and invoking Table 3. The presented confidence intervals are constructed using the $2.5 \%$ and $97.5 \%$ empirical quantiles. No confidence intervals were calculated for Gauss copulas as $\lambda_{Q T D}$ is zero per definition. The confidence bounds of the switching probabilities are obtained analogously. Initially estimated $\lambda_{Q T D}$-values are marked in bold.

\begin{tabular}{|c|c|c|c|c|c|}
\hline \multicolumn{2}{|c|}{ Index Pair } & \multicolumn{2}{|c|}{$\begin{array}{c}\text { “Normal” Regime } \lambda_{Q T D} \\
\text { with Simulation Mean and } \\
5 \% \text {-Confidence Interval }\end{array}$} & \multicolumn{2}{|c|}{$\begin{array}{c}\text { "Abnormal" Regime } \lambda_{Q T D} \text { anc } \\
\text { with Simulation Mean and } \\
5 \% \text {-Confidence Interval }\end{array}$} \\
\hline \multicolumn{6}{|c|}{ Asia } \\
\hline $\mathrm{Eq}-\mathrm{Eq}$ & NKY-HSI & 0.0000 & - & 0.4559 & $0.4547(0.3997,0.5077)$ \\
\hline $\mathrm{Eq}-\mathrm{Vol}$ & NKY-VNKY & 0.1059 & $0.1053(0.0281,0.1829)$ & 0.0001 & $0.0006(0.0000,0.0041)$ \\
\hline Eq-Vol & HSI-VHSI & 0.3788 & $0.3806(0.3225,0.4362)$ & 0.0050 & $0.0065(0.0001,0.0212)$ \\
\hline \multicolumn{6}{|c|}{ Europe } \\
\hline $\mathrm{Eq}-\mathrm{Eq}$ & DAX-SX5E & 0.0000 & - & 0.7653 & $0.7599(0.6985,0.8001)$ \\
\hline Eq-Vol & DAX-VDAX & 0.6531 & $0.6539(0.6233,0.6850)$ & 0.0000 & $0.0002(0.0000,0.0013)$ \\
\hline Eq-Vol & SX5E-VSX5E & 0.6105 & $0.6121(0.5755,0.6463)$ & 0.0000 & - \\
\hline \multicolumn{6}{|c|}{ USA } \\
\hline Eq-Vol & SPX-VIX & 0.6327 & $0.6325(0.5960,0.6637)$ & 0.0000 & $0.0000(0.0000,0.0003)$ \\
\hline \multicolumn{5}{|c|}{ Between continents } & $0.0898(0.0027,0.1925)$ \\
\hline $\mathrm{Eq}-\mathrm{Eq}$ & HSI-SX5E & 0.0000 & - & 0.2711 & $0.2715(0.2068,0.3401)$ \\
\hline $\mathrm{Eq}-\mathrm{Eq}$ & DAX-SPX & 0.0000 & - & 0.2409 & $0.2260(0.0376,0.3555)$ \\
\hline \multicolumn{6}{|c|}{ Switching probabilties } \\
\hline & Switching & 0.0684 & $0.0713(0.0432,0.1093)$ & 0.0723 & $0.0767(0.0465,0.1189)$ \\
\hline
\end{tabular}

We want to stress that for some index pairs, the results on QTD presented in Table 11 seem to be different to those obtained by the rolling window analysis presented in Table 8 . However this can be explained by the fact that for these specific pairs, the "abnormal" regime was only fitted in a certain percentage share of all windows while, in the final switching model, the whole time frame influences the parameters and therefore the fitted QTD of each pair.

\section{Summary}

In the present work, using an $R$-vine Markov-switching model with different, pre-defined $R$-vine structures, we aimed to find periods of "normal" and "abnormal" regimes within a data set consisting of North-American, European and Asian equity and volatility indices with an additional commodity index. We deliberately did not choose the usually applied wording of "non-crisis" and "crisis" as identified abnormality within the dependence structure does not necessarily go hand-in-hand with financial stress. After setting up sensible $R$-vine tree structures, we carried out an extensive rolling window analysis and drew the conclusion that regime changes mainly take place within the first trees. Estimating switching models for each continent individually and later on a joint global model shows the superiority of RV-MS setups over their corresponding static counterparts confirming the presence of global "normal" and "abnormal" regimes within the dependence structure of equity and implied volatility indices.

In fact, the joint model performed best supporting the hypothesis of the existence of global regime switches which can have important consequences for, e.g., portfolio diversification strategies. However, as this is beyond the scope of the present paper, we leave such a detailed analysis to future research. 
Additionally (as discussed in Section 2), in that context it might also be interesting to more deeply analyze the effects of time asynchronicity by methods like those proposed in [47].

Acknowledgments: The authors want to thank the editor and the anonymous reviewers for their helpful advice and various comments which lead to a real improvement of the present paper.

Author Contributions: All authors contributed equally to the paper.

Conflicts of Interest: The authors declare no conflict of interest.

\section{Appendix A. Model Summaries}

The following section contains all estimated model specifications. To compactly summarize each setup, we follow the $R$-vine notation of [68]: The $R$-vine-matrix $M^{\star}$ stores the specific nodes and edges for each tree (cf. Equation (4) and Figure 2 of [68]) while the copula-family-matrix $T^{\star}$ contains the respective bivariate copula types using the abbreviations of [40] (i.e., ' $1=\mathrm{Ga}^{\prime}$, ' $2=\mathrm{St}-t^{\prime}$, ' $4=\mathrm{Gu}^{\prime}$, ' $14=$ Gu180' ' $24=$ Gu90' and ' $34=$ Gu270'). Finally, the copula parameters are presented via $P_{1}^{\star}$ (and additionally $P_{2}^{\star}$ for the degrees of freedom in the Student- $t$ case) while the switching probabilities are denoted by $P$.

We want to stress that (1-dependent) is the only setup where a selection algorithm determined the $R$-Vine structure $M^{\star}$ and the bivariate copula families $T^{\star}$. For the non-switching regional models (Asia ${ }_{1}-\mathrm{Asia}_{4}$, Europe $_{1}-$ Europe $_{4}$ and $\mathrm{USA}_{1}-\mathrm{USA}_{2}$ ) as well as (2-dependent) the structure was manually chosen and only the copulas $T^{\star}$ were algorithmically selected based on maximum likelihood and AIC (cf. RVineCopSelect out of the VineCopula package by [40]). For the Markov-switching setups (Asia ${ }_{1, \mathbf{M S}}$, Europa $_{1, \mathbf{M S}}, \mathrm{USA}_{1, \mathrm{MS}}$ and (3-dependent-MS)) $M^{\star}$ and $T^{\star}$ for both regimes were manually determined. Parameters were always estimated and never fixed.

All "independence" model log-likelihoods were obtained via the sum of the regional setups. 
(1-dependent): $\quad$ 1-NKY 2-VNKY $\quad 3-H S I \quad 4-V H S I \quad$ 5-DAX $\quad$ 6-VDAX $\quad 7-S X 5 E \quad$ 8-VSX5E $\quad 9-S P X \quad 10-V I X \quad 11-B B C$

$$
\begin{aligned}
& M^{\star}=\left(\begin{array}{ccccccccccc}
2 & - & - & - & - & - & - & - & - & - & - \\
8 & 4 & - & - & - & - & - & - & - & - & - \\
6 & 8 & 1 & - & - & - & - & - & - & - & - \\
10 & 6 & 8 & 8 & - & - & - & - & - & - & - \\
9 & 10 & 6 & 3 & 6 & - & - & - & - & - & - \\
5 & 9 & 10 & 10 & 3 & 3 & - & - & - & - & - \\
11 & 5 & 9 & 9 & 10 & 10 & 11 & - & - & - & - \\
7 & 11 & 5 & 11 & 9 & 9 & 10 & 7 & - & - & - \\
4 & 7 & 11 & 7 & 11 & 5 & 9 & 10 & 5 & - & - \\
3 & 1 & 7 & 5 & 7 & 11 & 5 & 9 & 10 & 9 & - \\
1 & 3 & 3 & 6 & 5 & 7 & 7 & 5 & 9 & 10 & 10
\end{array}\right) \quad T^{\star}=\left(\begin{array}{cccccccccccccccccccc}
- \\
4 & - & - & - & - & - & - & - & - & - & - \\
2 & 4 & - & - & - & - & - & - & - & - & - \\
1 & 2 & 1 & - & - & - & - & - & - & - & - \\
14 & 1 & 1 & 24 & - & - & - & - & - & - & - \\
1 & 4 & 14 & 2 & 24 & - & - & - & - & - & - \\
1 & 2 & 34 & 1 & 2 & 4 & - & - & - & - & - \\
14 & 34 & 34 & 1 & 1 & 2 & 34 & - & - & - & - \\
1 & 34 & 14 & 2 & 1 & 2 & 2 & 1 & - & - & - \\
24 & 1 & 1 & 2 & 2 & 1 & 1 & 1 & 4 & - & - \\
24 & 24 & 2 & 2 & 2 & 2 & 2 & 2 & 2 & 34 & -
\end{array}\right)
\end{aligned}
$$

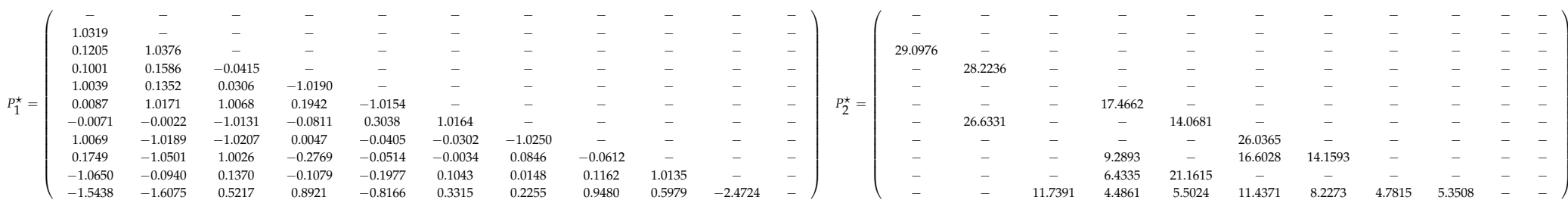


(2-dependent): $\quad$ 1-NKY $\quad$ 2-VNKY $\quad 3-H S I \quad 4-V H S I \quad$ 5-DAX $\quad 6$-VDAX $\quad 7-S X 5 E \quad$ 8-VSX5E 9 9-SPX $\quad 10-\mathrm{VIX} \quad$ 11-BBC

$$
\begin{aligned}
& M^{\star}=\left(\begin{array}{ccccccccccc}
6 & - & - & - & - & - & - & - & - & - & - \\
8 & 8 & - & - & - & - & - & - & - & - & - \\
2 & 10 & 10 & - & - & - & - & - & - & - & - \\
10 & 11 & 2 & 11 & - & - & - & - & - & - & - \\
11 & 9 & 4 & 2 & 9 & - & - & - & - & - & - \\
9 & 5 & 11 & 4 & 2 & 5 & - & - & - & - & - \\
4 & 2 & 1 & 1 & 4 & 2 & 7 & - & - & - & - \\
1 & 4 & 3 & 3 & 1 & 4 & 2 & 4 & - & - & - \\
3 & 1 & 7 & 7 & 3 & 1 & 4 & 2 & 3 & - & - \\
7 & 3 & 5 & 5 & 7 & 3 & 1 & 1 & 2 & 1 & - \\
5 & 7 & 9 & 9 & 5 & 7 & 3 & 3 & 1 & 2 & 2
\end{array}\right) \quad T^{\star}=\quad\left(\begin{array}{ccccccccccccccc}
- \\
2 & - & - & - & - & - & - & - & - & - & - & - \\
2 & 2 & - & - & - & - & - & - & - & - & - \\
2 & 1 & 1 & - & - & - & - & - & - & - & - \\
2 & 1 & 1 & 1 & - & - & - & - & - & - & - \\
1 & 2 & 24 & 24 & 14 & - & - & - & - & - & - \\
2 & 1 & 14 & 14 & 4 & 1 & - & - & - & - & - \\
1 & 2 & 4 & 1 & 24 & 2 & 14 & - & - & - & - \\
24 & 4 & 1 & 14 & 2 & 24 & 24 & 1 & - & - & - \\
2 & 24 & 4 & 14 & 1 & 2 & 1 & 1 & 34 & - & - \\
2 & 24 & 24 & 2 & 2 & 2 & 2 & 24 & 2 & 34 & -
\end{array}\right)
\end{aligned}
$$

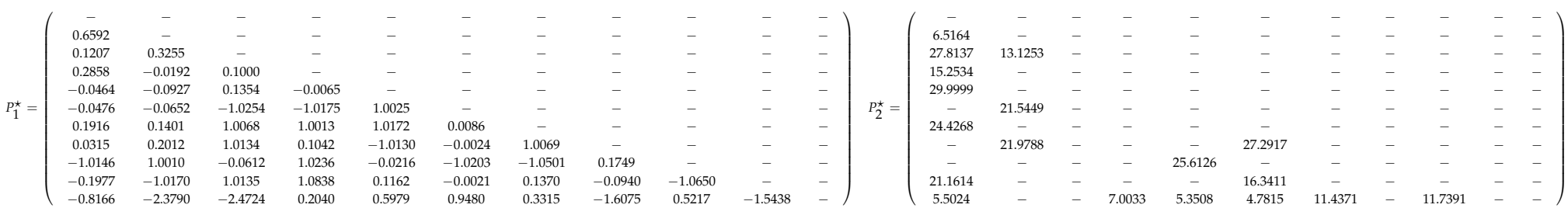


Econometrics 2017, 5, 3

30 of 38

Asia $_{1}$ : $\quad 1-\mathrm{NKY} \quad 2$-VNKY $\quad 3-\mathrm{HSI} \quad 4$-VHSI

$$
M^{\star}=\left(\begin{array}{cccc}
4 & - & - & - \\
2 & 2 & - & - \\
1 & 3 & 3 & - \\
3 & 1 & 1 & 1
\end{array}\right) \quad T^{\star}=\left(\begin{array}{cccc}
- & - & - & - \\
1 & - & - & - \\
1 & 24 & - & - \\
24 & 24 & 2 & -
\end{array}\right) \quad P_{1}^{\star}=\left(\begin{array}{cccc}
- & - & - & - \\
0.1749 & - & - & - \\
-0.0940 & -1.0650 & - & - \\
-1.6075 & -1.5438 & 0.5217 & -
\end{array}\right) P_{2}^{\star}=\left(\begin{array}{cccc}
- & - & - & - \\
- & - & - & - \\
- & - & - \\
- & 11.7391 & -
\end{array}\right)
$$

Asia 2 : 1-NKY 2-VNKY 3-HSI 4-VHSI

$$
M^{\star}=\left(\begin{array}{cccc}
3 & - & - & - \\
1 & 1 & - & - \\
2 & 4 & 4 & - \\
4 & 2 & 2 & 2
\end{array}\right) \quad T^{\star}=\left(\begin{array}{cccc}
- & - & - & - \\
2 & - & - & - \\
2 & 34 & - & - \\
34 & 34 & 4 & -
\end{array}\right) \quad P_{1}^{\star}=\left(\begin{array}{cccc}
- & - & - & - \\
0.3634 & - & - & - \\
-0.1741 & -1.1375 & - & - \\
-1.6075 & -1.5438 & 1.2745 & -
\end{array}\right) P_{2}^{\star}=\left(\begin{array}{cccc}
- & - \\
25.955 & - & - \\
- & - & - \\
28.6503 & - & - \\
- & - & -
\end{array}\right)
$$

Asia $_{3}$ : 1-NKY 2-VNKY 3-HSI 4-VHSI

$$
M^{\star}=\left(\begin{array}{cccc}
4 & - & - & - \\
2 & 2 & - & - \\
1 & 1 & 1 & - \\
3 & 3 & 3 & 3
\end{array}\right) \quad T^{\star}=\left(\begin{array}{cccc}
- & - & - & - \\
1 & - & - & - \\
1 & 2 & - & - \\
24 & 24 & 2 & -
\end{array}\right) \quad P_{1}^{\star}=\left(\begin{array}{cccc}
- & - & - & - \\
0.1710 & - & - & - \\
-0.0940 & -0.4097 & - & - \\
-1.6075 & -1.2713 & 0.5217 & -
\end{array}\right) P_{2}^{\star}=\left(\begin{array}{cccc}
- & - & - & - \\
- & - & - & - \\
- & 3.5933 & - & - \\
- & 11.7391 & -
\end{array}\right)
$$

Asia ${ }_{4}:$ 1-NKY 2-VNKY 3-HSI 4-VHSI

$$
M^{\star}=\left(\begin{array}{cccc}
4 & - & - & - \\
2 & 2 & - & - \\
3 & 3 & 3 & - \\
1 & 1 & 1 & 1
\end{array}\right) \quad T^{\star}=\left(\begin{array}{cccc}
- & - & - & - \\
1 & - & - & - \\
24 & 24 & - & - \\
24 & 24 & 2 & -
\end{array}\right) \quad P_{1}^{\star}=\left(\begin{array}{cccc}
- & - & - & - \\
0.1636 & - & - & - \\
-1.4269 & -1.0650 & - & - \\
-1.2774 & -1.5438 & 0.5217 & -
\end{array}\right) P_{2}^{\star}=\left(\begin{array}{cccc}
- & - & - & - \\
- & - & - & - \\
- & - & - \\
- & 11.7391 & -
\end{array}\right)
$$


Econometrics 2017, 5, 3

31 of 38

Europe $_{1}: \quad$ 1-DAX $\quad 2-V D A X \quad 3-S X 5 E \quad 4-V S X 5 E$

$M^{\star}=\left(\begin{array}{cccc}4 & - & - & - \\ 2 & 2 & - & - \\ 1 & 3 & 3 & - \\ 3 & 1 & 1 & 1\end{array}\right) \quad T^{\star}=\left(\begin{array}{cccc}- & - & - & - \\ 2 & - & - & - \\ 2 & 2 & - & - \\ 24 & 2 & 2 & -\end{array}\right) \quad P_{1}^{\star}=\left(\begin{array}{cccc}- & - & - & - \\ 0.7074 & - & - & - \\ -0.0619 & -0.1977 & - & - \\ -2.3790 & -0.8166 & 0.9480 & -\end{array}\right) \quad P_{2}^{\star}=\left(\begin{array}{cccc} & & - & - \\ - & - & - & - \\ 22.0738 & 21.1615 & - & - \\ - & 5.5024 & 4.7815 & -\end{array}\right)$

Europe $_{2}: \quad$ 1-DAX 2-VDAX 3-SX5E $\quad 4$-VSX5E

$M^{\star}=\left(\begin{array}{cccc}3 & - & - & - \\ 1 & 1 & - & - \\ 2 & 4 & 4 & - \\ 4 & 2 & 2 & 2\end{array}\right) \quad T^{\star}=\left(\begin{array}{cccc}- & - & - & - \\ 2 & - & - & - \\ 2 & 2 & - & - \\ 34 & 2 & 2 & -\end{array}\right) \quad P_{1}^{\star}=\left(\begin{array}{cccc}- & - & - & - \\ 0.8226 & - & - & - \\ -0.3768 & -0.1079 & - & - \\ -2.3790 & -0.8166 & 0.8921 & -\end{array}\right) \quad P_{2}^{\star}=\left(\begin{array}{cccc} & - & - & - \\ 7.7880 & - & - & - \\ 14.9738 & 6.435 & - & - \\ - & 5.5024 & 4.4861 & -\end{array}\right)$

Europe $3:$ 1-DAX 2-VDAX 3-SX5E 4-VSX5E

$M^{\star}=\left(\begin{array}{cccc}4 & - & - & - \\ 2 & 2 & - & - \\ 1 & 1 & 1 & - \\ 3 & 3 & 3 & 3\end{array}\right) \quad T^{\star}=\left(\begin{array}{cccc}- & - & - & - \\ 2 & - & - & - \\ 2 & 2 & - & - \\ 24 & 2 & 2 & -\end{array}\right) \quad P_{1}^{\star}=\left(\begin{array}{cccc}- & - & - & - \\ 0.0779 & - & - & - \\ -0.0619 & -0.2657 & - & - \\ -2.3790 & -0.0092 & 0.948 & -\end{array}\right) P_{2}^{\star}=\left(\begin{array}{cccc}- & - & - & - \\ 5.9311 & - & - & - \\ 22.0737 & 11.3322 & - & - \\ - & 6.6718 & 4.7815 & -\end{array}\right)$

Europe $_{4}: \quad$ 1-DAX $\quad$ 2-VDAX $3-S X 5 E \quad 4-V S X 5 E$

$M^{\star}=\left(\begin{array}{cccc}4 & - & - & - \\ 3 & 3 & - & - \\ 1 & 2 & 2 & - \\ 2 & 1 & 1 & 1\end{array}\right) \quad T^{\star}=\left(\begin{array}{cccc}- & - & - & - \\ 2 & - & - & - \\ 2 & 2 & - & - \\ 2 & 2 & 2 & -\end{array}\right) \quad P_{1}^{\star}=\left(\begin{array}{cccc}- & - & - & - \\ -0.2769 & - & - & - \\ -0.1079 & -0.1977 & - & - \\ 0.8921 & 0.9480 & -0.8166 & -\end{array}\right) \quad P_{2}^{\star}=\left(\begin{array}{cccc}- & - & - & - \\ 9.28933 & - & - & - \\ 6.4335 & 21.1615 & - \\ 4.4861 & 4.7815 & 5.5024 & -\end{array}\right)$

USA $_{1}$ : 1-SPX 2-VIX $\quad 3-B B C$

$M^{\star}=\left(\begin{array}{lll}3 & - & - \\ 2 & 2 & - \\ 1 & 1 & 1\end{array}\right) \quad T^{\star}=\left(\begin{array}{ccc}- & - & - \\ 34 & - & - \\ 2 & 24 & -\end{array}\right) \quad p_{1}^{*}=\left(\begin{array}{ccc}-1.206 & - & - \\ 0.204 & -24724 & -\end{array}\right) \quad p_{2}^{*}=\left(\begin{array}{ccc}- & - \\ 7.0033 & - & -\end{array}\right)$

USA $_{2}$ : 1-SPX $\quad 2$-VIX $\quad 3-\mathrm{BBC}$

$M^{\star}=\left(\begin{array}{ccc}3 & - & - \\ 1 & 1 & - \\ 2 & 2 & 2\end{array}\right) \quad T^{\star}=\left(\begin{array}{ccc}- & - & - \\ 4 & - & - \\ 34 & 34 & -\end{array}\right) \quad P_{1}^{\star}=\left(\begin{array}{ccc}- & - & - \\ 1.0595 & - & - \\ -1.1315 & -2.4724 & -\end{array}\right) P_{2}^{\star}=\left(\begin{array}{ccc}- & - & - \\ - & - & - \\ - & - & -\end{array}\right)$ 
Asia $_{1, \mathrm{MS}}: \quad 1-\mathrm{NKY} \quad 2$-VNKY $\quad 3$-HSI $\quad 4$-VHSI $\quad P=\left(\begin{array}{cc}0.9735 & 0.0554 \\ 0.0265 & 0.9446\end{array}\right)$

"normal" regime

$M^{\star}=\left(\begin{array}{cccc}4 & - & - & - \\ 2 & 2 & - & - \\ 1 & 3 & 3 & - \\ 3 & 1 & 1 & 1\end{array}\right) \quad T^{\star}=\left(\begin{array}{cccc}- & - & - & - \\ 1 & - & - & - \\ 1 & 24 & - & - \\ 24 & 24 & 1 & -\end{array}\right) \quad P_{1}^{\star}=\left(\begin{array}{cccc}- & - & - & - \\ 0.1462 & - & - & - \\ -0.0672 & -1.0429 & - \\ -1.4606 & -1.3081 & 0.4648 & -\end{array}\right) P_{2}^{\star}=\left(\begin{array}{cccc}- & - & - \\ - & - & - \\ - & - & - \\ - & - & -\end{array}\right)$

"abnormal" regime

$M^{\star}=\left(\begin{array}{cccc}4 & - & - & - \\ 2 & 2 & - & - \\ 1 & 3 & 3 & - \\ 3 & 1 & 1 & 1\end{array}\right) \quad T^{\star}=\left(\begin{array}{cccc}- & - & - & - \\ 1 & - & - & - \\ 1 & 24 & - & - \\ 2 & 2 & 14 & -\end{array}\right) \quad P_{1}^{\star}=\left(\begin{array}{cccc}- & - & - & - \\ 0.2025 & - & - & - \\ -0.1099 & -1.0921 & - & - \\ -0.7175 & -0.8065 & 1.6848 & -\end{array}\right) P_{2}^{\star}=\left(\begin{array}{cccc}- & - & - \\ - & - & - \\ - & - & - \\ 4.6616 & 8.3866 & - & -\end{array}\right)$

Europe $_{1, \text { MS }}: \quad$ 1-DAX $\quad$ 2-VDAX $\quad 3-S X 5 E \quad 4$-VSX5E $\quad P=\left(\begin{array}{ccc}0.9229 & 0.0973 \\ 0.0771 & 0.9027\end{array}\right)$

"normal" regime

$M^{\star}=\left(\begin{array}{cccc}4 & - & - & - \\ 2 & 2 & - & - \\ 1 & 3 & 3 & - \\ 3 & 1 & 1 & 1\end{array}\right) \quad T^{\star}=\left(\begin{array}{cccc}- & - & - & - \\ 2 & - & - & - \\ 34 & 1 & - & - \\ 24 & 24 & 1 & -\end{array}\right) \quad P_{1}^{\star}=\left(\begin{array}{cccc}- & - & - & - \\ 0.6907 & - & - & - \\ -1.0108 & -0.2245 & - & - \\ -2.0600 & -2.2610 & 0.9231 & -\end{array}\right) \quad P_{2}^{\star}=\left(\begin{array}{cccc}- & - & - \\ 8.115 & - & - \\ - & - & - \\ - & - & -\end{array}\right)$

"abnormal" regime

$M^{\star}=\left(\begin{array}{cccc}4 & - & - & - \\ 2 & 2 & - & - \\ 1 & 3 & 3 & - \\ 3 & 1 & 1 & 1\end{array}\right) \quad T^{\star}=\left(\begin{array}{cccc}- & - & - & - \\ 2 & - & - & - \\ 34 & 1 & - & - \\ 1 & 2 & 2 & -\end{array}\right) \quad P_{1}^{\star}=\left(\begin{array}{cccc}- & - & - & - \\ 0.7336 & - & - & - \\ -1.0469 & -0.1537 & - & - \\ -0.8836 & -0.8747 & 0.9756 & -\end{array}\right) P_{2}^{\star}=\left(\begin{array}{cccc}- & - & - \\ 4.4287 & - & - & - \\ - & - \\ - & 5.5657 & 29.9135 & -\end{array}\right)$ 
Econometrics 2017, 5, 3

33 of 38

USA $_{1, \mathrm{MS}}: \quad$ 1-SPX $\quad$ 2-VIX $\quad 3-\mathrm{BBC} \quad P=\left(\begin{array}{cc}0.9986 & 0.0025 \\ 0.0014 & 0.9975\end{array}\right)$

"normal" regime

$M^{\star}=\left(\begin{array}{ccc}3 & - & - \\ 2 & 2 & - \\ 1 & 1 & 1\end{array}\right) \quad T^{\star}=\left(\begin{array}{ccc}- & - & - \\ 1 & - & - \\ 1 & 24 & -\end{array}\right) \quad P_{1}^{\star}=\left(\begin{array}{ccc}- & - & - \\ -0.0423 & - & - \\ 0.0277 & -2.4687 & -\end{array}\right) P_{2}^{\star}=\left(\begin{array}{ccc}- & - & - \\ - & - \\ - & - & -\end{array}\right)$

“abnormal" regime

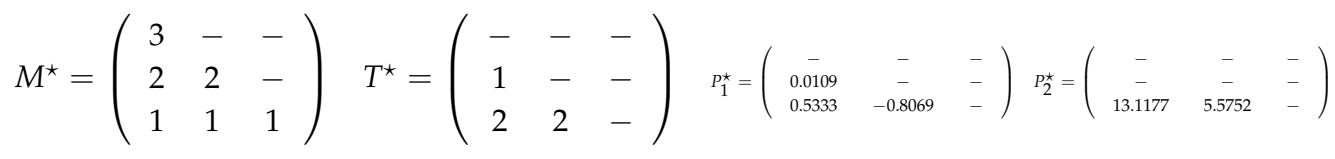


(3-dependent-MS): $\quad$ 1-NKY $\quad$ 2-VNKY $\quad 3-\mathrm{HSI} \quad$ 4-VHSI $\quad$ 5-DAX $\quad$ 6-VDAX $\quad 7-\mathrm{SX} 5 \mathrm{E} \quad$ 8-VSX5E $\quad$ 9-SPX $\quad$ 10-VIX $\quad$ 11-BBC $P=\left(\begin{array}{ll}0.9316 & 0.0723 \\ 0.0684 & 0.9277\end{array}\right)$

"normal" regime

$M^{\star}=\left(\begin{array}{ccccccccccc}6 & - & - & - & - & - & - & - & - & - & - \\ 8 & 8 & - & - & - & - & - & - & - & - & - \\ 2 & 10 & 10 & - & - & - & - & - & - & - & - \\ 10 & 11 & 2 & 11 & - & - & - & - & - & - & - \\ 11 & 9 & 4 & 2 & 9 & - & - & - & - & - & - \\ 9 & 5 & 11 & 4 & 2 & 5 & - & - & - & - & - \\ 4 & 2 & 1 & 1 & 4 & 2 & 7 & - & - & - & - \\ 1 & 4 & 3 & 3 & 1 & 4 & 2 & 4 & - & - & - \\ 3 & 1 & 7 & 7 & 3 & 1 & 4 & 2 & 3 & - & - \\ 7 & 3 & 5 & 5 & 7 & 3 & 1 & 1 & 2 & 1 & - \\ 5 & 7 & 9 & 9 & 5 & 7 & 3 & 3 & 1 & 2 & 2\end{array}\right) \quad T^{\star}=\left(\begin{array}{cccccccccccc}- \\ 2 & - & - & - & - & - & - & - & - & - & - \\ 2 & 2 & - & - & - & - & - & - & - & - & - \\ 2 & 1 & 1 & - & - & - & - & - & - & - & - \\ 2 & 1 & 1 & 1 & - & - & - & - & - & - & - \\ 1 & 2 & 24 & 24 & 14 & - & - & - & - & - & - \\ 2 & 1 & 14 & 14 & 4 & 1 & - & - & - & - & - \\ 1 & 2 & 4 & 1 & 24 & 2 & 14 & - & - & - & - \\ 24 & 4 & 1 & 14 & 2 & 24 & 24 & 1 & - & - & - \\ 2 & 24 & 4 & 14 & 1 & 2 & 1 & 1 & 34 & - & - \\ 24 & 24 & 24 & 1 & 4 & 1 & 1 & 24 & 1 & 24 & -\end{array}\right)$

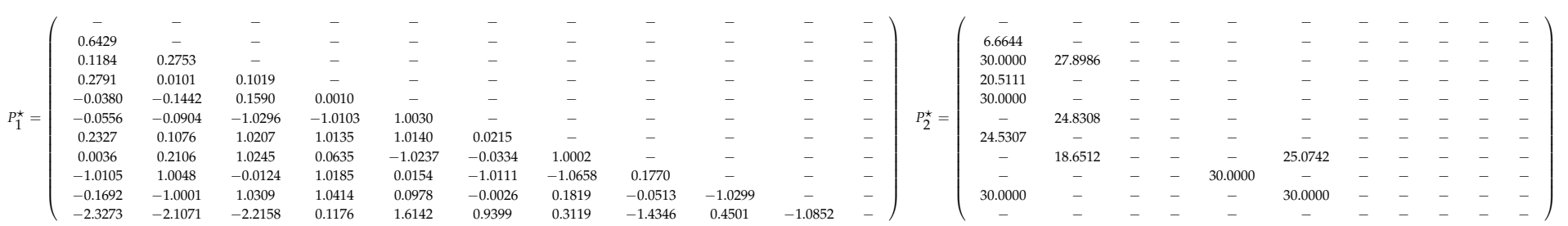


"abnormal" regime

$$
\begin{aligned}
& M^{\star}=\left(\begin{array}{ccccccccccc}
6 & - & - & - & - & - & - & - & - & - & - \\
8 & 8 & - & - & - & - & - & - & - & - & - \\
2 & 10 & 10 & - & - & - & - & - & - & - & - \\
10 & 11 & 2 & 11 & - & - & - & - & - & - & - \\
11 & 9 & 4 & 2 & 9 & - & - & - & - & - & - \\
9 & 5 & 11 & 4 & 2 & 5 & - & - & - & - & - \\
4 & 2 & 1 & 1 & 4 & 2 & 7 & - & - & - & - \\
1 & 4 & 3 & 3 & 1 & 4 & 2 & 4 & - & - & - \\
3 & 1 & 7 & 7 & 3 & 1 & 4 & 2 & 3 & - & - \\
7 & 3 & 5 & 5 & 7 & 3 & 1 & 1 & 2 & 1 & - \\
5 & 7 & 9 & 9 & 5 & 7 & 3 & 3 & 1 & 2 & 2
\end{array}\right) \quad T^{\star}=\left(\begin{array}{ccccccccccc}
- & - & - & - & - & - & - & - & - & - & - \\
2 & - & - & - & - & - & - & - & - & - & - \\
2 & 2 & - & - & - & - & - & - & - & - & - \\
2 & 1 & 1 & - & - & - & - & - & - & - & - \\
2 & 1 & 1 & 1 & - & - & - & - & - & - & - \\
1 & 2 & 24 & 24 & 14 & - & - & - & - & - & - \\
2 & 1 & 14 & 14 & 4 & 1 & - & - & - & - & - \\
1 & 2 & 4 & 1 & 24 & 2 & 14 & - & - & - & - \\
24 & 4 & 1 & 14 & 2 & 24 & 24 & 1 & - & - & - \\
2 & 24 & 4 & 14 & 1 & 2 & 1 & 1 & 34 & - & - \\
2 & 1 & 2 & 2 & 2 & 2 & 14 & 2 & 14 & 2 & -
\end{array}\right)
\end{aligned}
$$

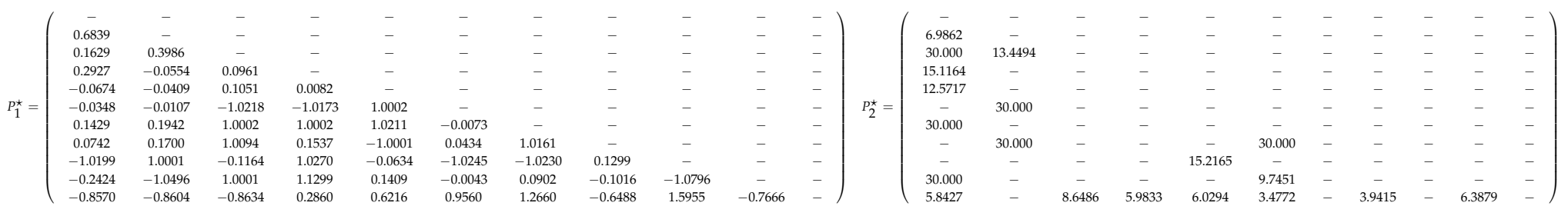




\section{References}

1. Brenner, M.; Galai, D. New Financial Instruments for Hedge Changes in Volatility. Financial Anal. J. 1989, 45, 61-65.

2. Whaley, R.E. Derivatives on market volatility: Hedging tools long overdue. J. Deriv. 1993, 1, 71-84.

3. Fleming, J.; Ostdiek, B.; Whaley, R.E. Predicting stock market volatility: A new measure. J. Futures Mark. 1995, 15, 265-302.

4. Fleming, J. The quality of market volatility forecasts implied by S \& P 100 index option prices. J. Empir. Finance 1998, 5, 317-345.

5. Christensen, B.J.; Prabhala, N.R. The relation between implied and realized volatility. J. Financial Econ. 1998, 50, 125-150.

6. Grünbichler, A.; Longstaff, F.A. Valuing futures and options on volatility. J. Bank. Finance 1996, 20, 985-1001.

7. Hao, J.; Zhang, J.E. GARCH Option Pricing Models, the CBOE VIX, and Variance Risk Premium. J. Financial Econom. 2012, 11, 556-580.

8. Kanniainen, J.; Lin, B.; Yang, H. Estimating and using GARCH models with VIX data for option valuation. J. Bank. Finance 2014, 43, 200-211.

9. Black, F. Studies of Stock Price Volatility Changes. In Proceedings of the Business and Economics Section of the American Statistical Association; American Statistical Association: Washington, DC, USA, 1976; pp. 177-181.

10. Allen, D.E.; Singh, A.K.; Powell, R.J.; McAleer, M.; Taylor, J.W.; Thomas, L.C. Return-Volatility Relationship: Insights from Linear and Non-Linear Quantile Regression. 2013. Available online: http://papers.ssrn.com/ sol3/papers.cfm?abstract_id=2253685 (accessed on 18 March 2016).

11. Ning, C.; Xu, D.; Wirjanto, T.S. Is volatility clustering of asset returns asymmetric? J. Bank. Finance 2015, 52, 62-76.

12. Banerjee, P.S.; Doran, J.S.; Peterson, D.R. Implied volatility and future portfolio returns. J. Bank. Finance 2007, 31, 3183-3199.

13. Rubbaniy, G.; Asmerom, R.; Rizvi, S.K.A.; Naqvi, B. Do fear indices help predict stock returns? Quant. Finance 2014, 14, 831-847.

14. Egbers, T.; Swinkels, L. Can implied volatility predict returns on the currency carry trade? J. Bank. Finance 2015, 59, 14-26.

15. Mele, A.; Obayashi, Y.; Shalen, C. Rate fears gauges and the dynamics of fixed income and equity volatilities. J. Bank. Finance 2015, 52, 256-265.

16. Dotsis, G.; Psychoyios, D.; Skiadopoulos, G. An empirical comparison of continuous-time models of implied volatility indices. J. Bank. Finance 2007, 31, 3584-3603.

17. Fernandes, M.; Medeiros, M.C.; Scharth, M. Modeling and predicting the CBOE market volatility index. J. Bank. Finance 2014, 40,1-10.

18. Aloui, C. Price and volatility spillovers between exchange rates and stock indexes for the pre- and post-euro period. Quant. Finance 2007, 7, 669-685.

19. Peng, Y.; Ng, W.L. Analysing Financial Contagion and Asymmetric Market Dependence with Volatility Indices via Copulas. Ann. Finance 2012, 8, 49-74.

20. Antonakakis, N.; Chatziantoniou, I.; Filis, G. Dynamic co-movements of stock market returns, implied volatility and policy uncertainty. Econ. Lett. 2013, 120, 87-92.

21. Jung, R.C.; Maderitsch, R. Structural breaks in volatility spillovers between international financial markets: Contagion or mere interdependence? J. Bank. Finance 2014, 47, 331-342.

22. Nomikos, N.; Salvador, E. The role of volatility regimes on volatility transmission patterns. Quant. Finance 2013, 14, 1-13.

23. Strohsal, T.; Weber, E. Time-varying international stock market interaction and the identification of volatility signals. J. Bank. Finance 2015, 56, 28-36.

24. Chollete, L.; de la Peña, V.; Lu, C.C. International diversification: A copula approach. J. Bank. Finance 2011, 35, 403-417.

25. Patton, A.J. Copula-Based Models for Financial Time Series. In Handbook of Financial Time Series; Springer: New York, NY, USA, 2009; pp. 767-785.

26. Hull, J.C.; White, A.D. Valuing Credit Derivatives Using an Implied Copula Approach. J. Deriv. 2006, $14,8-28$. 
27. Fermanian, J.D.; Vigneron, O. Pricing and hedging basket credit derivatives in the Gaussian copula. Risk 2010, 23, 92-96.

28. Rodriguez, J.C. Measuring financial contagion: A Copula approach. J. Empir. Finance 2007, 14, 401-423.

29. Okimoto, T. New Evidence of Asymmetric Dependence Structures in International Equity Markets. J. Financial Quant. Anal. 2008, 43, 787-815.

30. Arakelian, V.; Dellaportas, P. Contagion determination via copula and volatility threshold models. Quant. Finance 2012, 12, 295-310.

31. Bartram, S.M.; Wang, Y.H. European financial market dependence: An industry analysis. J. Bank. Finance 2015, 59, 146-163.

32. Salazar, Y.; Ng, W.L. Nonparametric Tail Copula Estimation: An Application to Stock and Volatility Index Returns. Commun. Stat. Simul. Comput. 2013, 42, 613-635.

33. Kasch, M.; Caporin, M. Volatility Threshold Dynamic Conditional Correlations: An International Analysis. J. Financial Econom. 2013, 11, 706-742.

34. Hamilton, J.D. A New Approach to the Economic Analysis of Nonstationary Time Series and the Business Cycle. Econometrica 1989, 57, 357-384.

35. Chollete, L.; Heinen, A.; Valdesogo, A. Modeling International Financial Returns with a Multivariate Regime-switching Copula. J. Financial Econom. 2009, 7, 437-480.

36. Da Silva Filhoa, O.C.; Ziegelmann, F.A.; Dueker, M.J. Modeling dependence dynamics through copulas with regime switching. Insur. Math. Econ. 2012, 50, 346-356.

37. Wang, Y.C.; Wu, J.L.; Lai, Y.H. A revisit to the dependence structure between the stock and foreign exchange markets: A dependence-switching copula approach. J. Bank. Finance 2013, 37, 1706-1719.

38. Stöber, J.; Czado, C. Regime Switches in the Dependence Structure of Multivariate Financial Data. Comput. Stat. Data Anal. 2014, 76, 672-686.

39. Ghalanos, A. Rugarch: Univariate GARCH models.; R package version 1.3-6.; 2015. Available online: https:/ / cran.r-project.org/web/packages/rugarch/rugarch.pdf (accessed on 18 March 2016).

40. Schepsmeier, U.; Stöber, J.; Brechmann, E.; Gräler, B.; Nagler, T.; Erhardt, T. VineCopula: Statistical Inference of Vine Copulas; R Package Version; Technische Universität München: München, Germany, 2015.

41. Epps, T.W. Comovements in Stock Prices in the Very Short Run. J. Am. Stat. Assoc. 1979, 74, 291-298.

42. Scholes, M.; Williams, J. Estimating betas from nonsynchronous data. J. Financial Econ. 1977, 5, 309-327.

43. Martens, M.; Poon, S.H. Returns synchronization and daily correlation dynamics between international stock markets. J. Bank. Finance 2001, 25, 1805-1827.

44. Hayashi, T.; Yoshida, N. On covariance estimation of non-synchronously observed diffusion processes. Bernoulli 2005, 11, 359-379.

45. Levy, A.; Lieberman, O. Overreaction of country ETFs to US market returns: Intraday vs. daily horizons and the role of synchronized trading. J. Bank. Finance 2013, 37, 1412-1421.

46. Baur, D.G. Financial contagion and the real economy. J. Bank. Finance 2012, 36, 2680-2692.

47. Burns, P.; Engle, R.; Mezrich, J. Correlations and volatilities of asynchronous data. Rev. Deriv. Res. 1998, 5, 7-18.

48. Aguilar, M.; Hill, J.B. Robust score and portmanteau tests of volatility spillover. J. Econom. 2015, 184, 37-61.

49. Audrino, F.; Bühlmann, P. Synchronizing Multivariate Financial Time Series. J. Risk 2004, 6, 81-106.

50. Beil, M. Modeling Dependencies among Financial Asset Returns Using Copulas. Master's Thesis, Technische Universität München, München, Germany, 2013.

51. Joe, H. Multivariate Models and Dependence Concepts; Chapman and Hall: London, UK, 1997.

52. Nelsen, R.B. An Introduction to Copulas; Springer: New York, NY, USA, 2006.

53. Kurowicka, D.; Cooke, R. Uncertainty Analysis with High Dimensional Dependence Modelling; Wiley Series in Probability and Statistics: New York, NY, USA, 2006.

54. Kurowicka, D.; Joe, H. Dependence Modeling-Handbook on Vine Copulae; World Scientific: London, UK, 2010.

55. Stöber, J.; Czado, C. Sampling Pair Copula Constructions with Applications to Mathematical Finance. In Simulating Copulas: Stochastic Models, Sampling Algorithms, and Applications; Series in Quantitative Finance; Mai, J.F., Scherer, M., Eds.; World Scientific: London, UK, 2012; Volume 4.

56. Joe, H. Dependence Modeling with Copulas; Monographs on Statistics and Applied Probability, CRC Press: Boca Raton, FL, USA, 2014. 
57. Sklar, M. Fonctions de répartition à $n$ dimensions et leurs marges; Publications de l'Institut de Statistique de L'Universite de Paris: Paris, France, 1959; Volume 8, pp. 229-231.

58. Bedford, T.; Cooke, R. Probability density decomposition for conditionally dependent random variables modeled by vines. Ann. Math. Artif. Intell. 2001, 32, 245-268.

59. Bedford, T.; Cooke, R. Vines-A new graphical model for dependent random variables. Ann. Stat. 2002, 30, 1031-1068.

60. Joe, H. Families of $m$-variate Distributions with Given Margins and $m(m-1) / 2$ Bivariate Dependence Parameters. Lect. Notes-Monogr. Ser. 1996, 28, 120-141.

61. Aas, K.; Czado, C.; Frigessi, A.; Bakken, H. Pair-copula Constructions of Multiple Dependence. Insur. Math. Econ. 2009, 44, 182-198.

62. Czado, C. Pair-copula constructions of multivariate copulas. In Copula Theory and Its Applications; Lecture Notes in Statistics; Jaworski, P., Durante, F., Härdle, W., Rychlik, T., Eds.; Springer: Berlin, Germany, 2010.

63. Hobæk Haff, I.; Aas, K.; Frigessi, A. On the simplified pair-copula construction-Simply useful or too simplistic? J. Multivar. Anal. 2010, 101, 1296-1310.

64. Acar, E.F.; Genest, C.; Nešlehová, J. Beyond simplified pair-copula constructions. J. Multivar. Anal. 2012, 110, 74-90.

65. Stöber, J.; Joe, H.; Czado, C. Simplified pair copula constructions-Limitations and extensions. J. Multivar. Anal. 2013, 119, 101-118.

66. Thulasiraman, K.; Swamy, M. Graphs: Theory and Algorithms; John Wiley \& Sons: New York, NY, USA, 1992.

67. Bernard, C.; Czado, C. Conditional quantiles and tail dependence. J. Multivar. Anal. 2015, 138, $104-126$.

68. Dissmann, J.; Brechmann, E.C.; Czado, C.; Kurowicka, D. Selecting and estimating regular vine copulae and application to financial returns. Comput. Stat. Data Anal. 2013, 59, 52-69.

69. Kim, C.J.; Nelson, C.R. State-space Models with Regime Switching; MIT Press: Cambridge, MA, USA, 2006.

70. Wu, C.F.J. On the convergence properties of the EM algorithm. Ann. Stat. 1983, 11, 95-103.

71. Leroux, B.G. Maximum-likelihood estimation for hidden Markov models. Stoch. Process. Their Appl. 1992, 40, 127-143.

72. Christoffersen, P.; Errunza, V.; Jacobs, K.; Langlois, H. Is the potential for international diversifcation disappearing? A dynamic copula approach. Rev. Financial Stud. 2012, 25, 3711-3751.

73. Okimoto, T. Asymmetric increasing trends in dependence in international equity markets. J. Bank. Finance 2014, 138, 219-232.

74. French, K.R.; Schwert, G.W.; Stambaugh, R.F. Expected stock returns and volatility. J. Financial Econ. 1987, 19, 3-29.

(C) 2017 by the authors; licensee MDPI, Basel, Switzerland. This article is an open access article distributed under the terms and conditions of the Creative Commons Attribution (CC-BY) license (http:/ / creativecommons.org/licenses/by/4.0/). 